\title{
ON THE ASYMPTOTICALLY LINEAR HÉNON PROBLEM
}

\author{
ANNA LISA AMADORI ${ }^{\dagger}$
}

\begin{abstract}
In this paper we consider the Hénon problem in the ball with Dirichlet boundary conditions. We study the asymptotic profile of radial solutions and then deduce the exact computation of their Morse index when the exponent $p$ is close to 1 . Next we focus on the planar case and describe the asymptotic profile of some solutions which minimize the energy among functions which are invariant for reflection and rotations of a given angle $2 \pi / n$. By considerations based on the Morse index we see that, depending on the values of $\alpha$ and $n$, such least energy solutions can be radial, or nonradial and different one from another.
\end{abstract}

Keywords: nodal, least energy, radial and non-radial solutions; asymptotic profile; Morse index.

AMS Subject Classifications: 35J91, 35B06, 35B40, 35P05

\section{INTRODUCTION}

This paper investigates the Hénon problem

$$
\begin{cases}-\Delta u=|x|^{\alpha}|u|^{p-1} u & \text { in } B, \\ u=0 & \text { on } \partial B,\end{cases}
$$

where $\alpha>0, B$ stands for the unitary ball in $\mathbb{R}^{N}$ with $N \geq 2$, and the exponent $p$ is next to one.

It is well known that, for $\alpha>0$ fixed, the Hénon problem (1.1) admits solutions, and in particular radial solutions, for every $p>1$ in dimension $N=2$, and for every $p \in\left(1, \frac{N+2+2 \alpha}{N-2}\right)$ in dimension $N \geq 3$. In that range of existence, for any given $m \geq 1$ there is exactly one couple of radial solutions of (1.1) which have exactly $m$ nodal zones, they are classical solutions and they are one the opposite of the other (see 24, [10, 25]). It is well known that (1.1) has also nonradial positive solutions, and the literature on this subject is rich. First [28] showed that for every value of $p$ below the Sobolev critical exponent, then the minimal energy solution is nonradial when $\alpha$ is large enough. Next nonradial solutions have been produced in a number of works by various techniques: we mention among others [26], 14, 21 based on Lyapunov-Schmidt reduction, 27, 7] relying on different constrained minimization, and [3] using bifurcation. Nevertheless it is worth mentioning that for $\alpha>0$ fixed there is a neighborhood of $p=1$ (clearly depending by $\alpha$ ) where the only positive solution to (1.1) is the radial one, see 3. Theorem 3.1].

Concerning nodal solutions, considerations based on the Morse index yield that the minimal energy solution is nonradial for every $\alpha>0$ and $p$ in the existence range. Indeed the minimal energy nodal solution has Morse index 2 by [8], while the Morse index of nodal radial solutions is greater than 4 , see [5]. The same phenomenon was already pointed out in [1, concerning the Lane Emden problem. In dimension $N=2$ and for large values of $p$ sign-changing multipeak solutions have been produced in [30. In the same range [7] proved a multiplicity result by constructing least energy nodal solutions in suitable symmetric spaces and comparing their Morse index with the one of radial solutions. In higher dimension

Date: June 4, 2019.

The author is member of the Gruppo Nazionale per l'Analisi Matematica, la Probabilità e le loro Applicazioni (GNAMPA) of the Istituto Nazionale di Alta Matematica (INdAM). . 
another recent paper 22 produced nonradial solutions by bifurcation w.r.t. the parameter $\alpha$, by taking advantage from the fact that the Morse index of radial solutions goes to infinity. In this perspective knowing the exact Morse index is an essential step in producing nonradial solutions. Its value has been computed when the parameter $\alpha$ is large in [23, and when the parameter $p$ is close to the supremum of the existence range in [6] (in dimension $N \geq 3$ ) and in [7] (in dimension $N=2$ ).

Here we describe the asymptotic profile of radial solutions and compute the exact value of their Morse index for $p$ close to 1 , and we use it to obtain, for any given value of $\alpha$, nonradial solutions which live in a neighborhood of $p=1$, and which anyway preserve some rotational symmetry. In doing this we also describe the asymptotic profile of such noradial solutions.

But let us go in order. The papers [11] and 20] investigated the Lane-Emden problem settled in any domain $\Omega$, when $p$ approaches 1 , and described the behaviour of the solutions in terms of the eigenvalues of the Laplace operator on $\Omega$, i.e.

$$
\begin{cases}-\Delta \omega=\mu \omega & \text { in } \Omega, \\ \omega=0 & \text { on } \partial \Omega,\end{cases}
$$

showing, among other things, that any sequence of solutions whose norm in $L^{2}(\Omega)$ is suitably bounded converges (up to a subsequence) to an eigenfunction of (1.2). The radial setting is clearly simpler, and one can see that any radial solutions satisfies the $L^{2}$ bound as $p$ is next to 1 , and a sequence of radial solutions with $m$ nodal zones converges to the $m^{\text {th }}$ radial eigenfunction of (1.2), which is simple and is nothing else that a Bessel function. Analogous result holds for the Hénon problem (1.1), provided that the eigenvalue problem for the Laplacian is replaced by the weighted eigenvalue problem

$$
\begin{cases}-\Delta \omega=\mu|x|^{\alpha} \omega & \text { in } B, \\ \omega=0 & \text { on } \partial B,\end{cases}
$$

which clearly reduces to (1.2) when $\alpha=0$ and $\Omega$ is a ball. Our first result, presented in Section 3, stands in computing the eigenvalues and eigenfunctions of (1.3) and describing the asymptotic behaviour of radial solutions as $p \rightarrow 1$.

We denote by $\Gamma$ the Gamma-function, by $\mathcal{J}_{\beta}$ the Bessel function of first kind defined as

$$
\mathcal{J}_{\beta}(r)=r^{\beta} \sum_{k=0}^{+\infty} \frac{(-1)^{k}}{k ! \Gamma(k+1+\beta)}\left(\frac{r}{2}\right)^{2 k}, \quad r \geq 0,
$$

and by $z_{n}(\beta)$ the sequence of its positive zeros. We will prove that

Theorem 1.1. Let $u_{p}$ be a radial solution to (1.1) with $m$ nodal zones for $\alpha \geq 0$. When $p \rightarrow 1$ we have

$$
\begin{aligned}
\left\|u_{p}\right\|_{\infty}^{\frac{p-1}{2}} & \rightarrow \frac{2+\alpha}{2} z_{m}\left(\frac{N-2}{2+\alpha}\right), \\
\frac{u_{p}(x)}{\left\|u_{p}\right\|_{\infty}} & \rightarrow \pm \Gamma\left(\frac{N+\alpha}{2+\alpha}\right)|x|^{-\frac{N-2}{2}} \mathcal{J}_{\frac{N-2}{2+\alpha}}\left(z_{m}\left(\frac{N-2}{2+\alpha}\right)|x|^{\frac{2+\alpha}{2}}\right) \text { in } C^{2}\left(B_{1}\right) .
\end{aligned}
$$

Moreover denoting by $0<r_{1, p}<\ldots r_{m, p}=1$ the nodal radii of $u_{p}$ we have

$$
r_{i, p} \rightarrow\left(\frac{z_{i}\left(\frac{N-2}{2+\alpha}\right)}{z_{m}\left(\frac{N-2}{2+\alpha}\right)}\right)^{\frac{2}{2+\alpha}} \quad \text { as } i=1, \ldots m-1 .
$$

Next we exploit the characterization of the Morse index in terms of a singular SturmLiouville problem given in 44 and recalled here in Subsection 3.1. Thanks to the convergence established in Theorem [1.1] we are able to pass to the limit also in that singular problem and compute the Morse index of $u_{p}$ in a right neighborhood of $p=1$. To state the related result some more notation is needed. Since the map $\beta \mapsto z_{i}(\beta)$ is continuous and increasing, 
for every integer $m$ fixed there exist $\beta_{i}=\beta_{i}(\alpha, N)>0$ such that $z_{i}\left(\beta_{i}\right)$ (the $i^{\text {th }}$ zero of the Bessel function $\mathcal{J}_{\beta_{i}}$ ) coincides with $z_{m}\left(\frac{N-2}{2+\alpha}\right)$ (the $m^{t h}$ zero of $\mathcal{J}_{\frac{N-2}{2+\alpha}}$ ). Next we write

$$
N_{j}:= \begin{cases}1 & \text { when } j=0 \\ \frac{(N+2 j-2)(N+j-3) !}{(N-2) ! j !} & \text { when } j \geq 1\end{cases}
$$

for the multiplicity of the eigenvalue $\lambda_{j}:=j(N+j-2)$ of the Laplace-Beltrami operator in the sphere $\mathbb{S}_{N-1}$, and $\lceil s\rceil=\min \{n \in \mathbb{Z}: n \geq s\}$ for the ceiling function.

Theorem 1.2. Let $u_{p}$ be a radial solution to (1.1) with $m$ nodal zones. For every $\alpha \geq 0$ there is $\bar{p}=\bar{p}(\alpha)>1$ such that for $p \in(1, \bar{p})$ the Morse index of $u_{p}$ is given by

$$
m\left(u_{p}\right)=1+\sum_{i=1}^{m-1} \sum_{j=0}^{\left\lceil\frac{(2+\alpha) \beta_{i}-N}{2}\right\rceil} N_{j}
$$

if $\alpha \neq \alpha_{\ell, n}=(2 n+N-2) / \beta_{\ell}-2($ as $\ell=1, \ldots m-1, n \in \mathbb{N})$. Otherwise if $\alpha=\alpha_{\ell, n}$ the Morse index is estimated by

$$
1+\sum_{i=1}^{m-1} \sum_{j=0}^{\left\lceil\frac{(2+\alpha) \beta_{i}-N}{2}\right\rceil} N_{j} \leq m\left(u_{p}\right) \leq 1+\sum_{i=1}^{m-1} \sum_{j=0}^{\left\lceil\frac{(2+\alpha) \beta_{i}-N}{2}\right\rceil} N_{j}+\sum_{\ell} \frac{N_{(2+\alpha) \beta_{\ell}-N}}{2}+1
$$

In the particular case of positive solutions Theorem 1.2 recovers that $m\left(u_{p}\right)=1$, which is clearly true for the positive solution to the Lane-Emden equation (whose Morse index is equal to 1 for any value of the parameter $p$ ), and was already proved in [3] for the Hénon equation in dimension $N \geq 3$. Coming to nodal solutions, the formula (1.7) is not totally explicit since the law $\beta \mapsto z_{i}(\beta)$ is not known. However the value of $z_{i}(\beta)$ can be computed by a numerical procedure (for instance by the command besselzero in MatLab), and by a dichotomy argument the approximated values of $\beta_{i}^{m}$ can be deduced.

For the Lane-Emden equation $(\alpha=0)$ the numerical approximation suggests that $2(m-$ i) $-1<\beta_{i}-\frac{N}{2}<2(m-i)$, so that (1.7) becomes

$$
m\left(u_{p}\right)=m+\sum_{i=1}^{m}(1+m-i)\left(N_{2 i-1}+N_{2 i}\right)
$$

which simplifies into

$$
m\left(u_{p}\right)=m(2 m-1)
$$

in dimension $N=2$.

In the plane the approximation procedure is elementary also for $\alpha>0$, because the baseline Bessel function is $\mathcal{J}_{0}$, whose zeros are tabulated. Of particular interest is the case of the radial solution with 2 nodal zones, which is the least energy nodal radial solution (see [9]) and will be denoted by $u_{p}^{*}$ in the following. In that case formula (1.7) becomes

$$
m\left(u_{p}^{*}\right)=2\left\lceil\frac{2+\alpha}{2} \beta\right\rceil, \quad \text { with } \beta \approx 2,305 .
$$

In particular the least energy nodal radial solution to the Lane-Emden equation $(\alpha=0)$ has Morse index 6, as already noticed in [19. For small positive values of $\alpha$ the Morse index remains 6 , while there is a sequence of critical values $\alpha_{n}=2(n / \beta-1)$ where the asymptotic Morse index increases. This phenomenon, which is enlightened here for the first time, suggests that the structure of the set of the solutions to (1.1) changes in correspondence of these values of $\alpha$, for $p$ arbitrarily close to 1 .

To explore this issue further we focus onto the so called $n$-invariant solutions, introduced in 19 in the Lane-Emden case, and studied also in 7] in the Hénon case (for large values of $p$ ). We say that a function defined on the 2 -dimensional unitary ball $B$ is $n$-invariant if it 
is invariant for reflection across the horizontal axis and for rotations of an angle $2 \pi / n$, and we denote by $H_{0, n}^{1}$ the subspace of $H_{0}^{1}(B)$ made up by $n$-invariant functions, i.e. in polar coordinates

$$
H_{0, n}^{1}:=\left\{u \in H_{0}^{1}(B): u(r, \theta) \text { is even and } 2 \pi / n \text { periodic w.r.t. } \theta \text {, for every } r \in(0,1)\right\} \text {. }
$$

A by now standard compactness argument in the respective nodal Nehari manifold (see [8]) produces, for every integer $n$ and $p>1$, a nodal solution to (1.1) which is $n$-invariant, and minimizes the associated energy among $n$-invariant functions. We denote by $U_{p, n}$ such $n$-invariant nodal least energy solution. In particular $U_{p, 1}$ coincides with the least energy nodal solution, thanks to the symmetry result in 9], and so it is known that it is nonradial for every value of $p$. Coming to $n \geq 2$, it is not known if $U_{p, n}$ are radial or not, neither if they are distinct one from another. For instance in the Lane-Emden case [19] showed that for $p$ close to $1 U_{p, n}$ are nonradial as $n=1,2$, while they are radial and coincide with $u_{p}^{*}$ for $n \geq 3$.

To find an answer to this questions we investigate the asymptotic profile of $U_{p, n}$ for $p$ close to 1 , proving that

Theorem 1.3. Let $U_{p, n}$ be a n-invariant least energy nodal solution to (1.1) in dimension $N=2$. As $p \rightarrow 1$ we have

$$
\begin{aligned}
& \left\|U_{p, n}\right\|_{\infty}^{p-1} \longrightarrow\left(\frac{2+\alpha}{2} z_{1}\left(\frac{2 n}{2+\alpha}\right)\right)^{2} \\
& \frac{U_{p, n}(r, \theta)}{\left\|U_{p, n}\right\|_{\infty}} \longrightarrow \pm \frac{1}{\left\|\mathcal{J}_{\frac{2 n}{2+\alpha}}\right\|_{\infty}} \mathcal{J}_{\frac{2 n}{2+\alpha}}\left(z_{1}\left(\frac{2 n}{2+\alpha}\right) r^{\frac{2+\alpha}{2}}\right) \cos (n \theta) .
\end{aligned}
$$

for $n<\frac{2+\alpha}{2} \beta$, and

$$
\begin{aligned}
& \left\|U_{p, n}\right\|_{\infty}^{p-1} \longrightarrow\left(\frac{2+\alpha}{2} z_{2}(0)\right)^{2}, \\
& \frac{U_{p, n}(r, \theta)}{\left\|U_{p, n}\right\|_{\infty}} \longrightarrow \pm \mathcal{J}_{0}\left(z_{2}(0) r^{\frac{2+\alpha}{2}}\right) .
\end{aligned}
$$

for $n>\frac{2+\alpha}{2} \beta$.

Here $\beta \approx 2,305$ is the same number as in 1.9 .

The proof of Theorem 1.3. reported in Subsection 4.1, is quite long and involved. First, by a refined blow-up technique relying on the Morse index in the space $H_{0, n}^{1}$, we establish an estimate which ensures that $U_{p, n}$ converges to an eigenfunction of (1.3). Next, taking advantage by the minimality of $U_{p, n}$, we see that its limit must be the second eigenfunctions of (1.3) in the space $H_{0, n}^{1}$. Here is the point where the number $\frac{2+\alpha}{2} \beta$ comes into play, because it is the threeshold under which the second eigenfunction in $H_{0, n}^{1}$ is nonradial.

Starting from the asymptotic description in Theorem 1.3 we can see that, for $p$ close to one, $U_{p, n}$ are nonradial and distinct for $n=1, \ldots\left\lceil\frac{2+\alpha}{2} \beta-1\right\rceil$, while they coincide with the radial nodal least energy solution $u_{p}^{*}$ for $n>\frac{2+\alpha}{2} \beta$, thus obtaining the following multiplicity result.

Theorem 1.4. In dimension $N=2$ there exists $\bar{p}=\bar{p}(\alpha)>1$ such that (1.1) has $\left\lceil\frac{2+\alpha}{2} \beta-1\right\rceil$ distinct nodal nonradial solutions for every $p \in(1, \bar{p}(\alpha))$.

The solutions are distinct meaning that they cannot be obtained from each other by reflection or rotation, and of course they are not one the opposite of the other. When $\alpha=0$ Theorem 1.4 provides 2 solutions and gives back the multiplicity result in [19], from which the present one borrows many ideas.

Finally we compare Theorem [1.4 with [7, Theorem 1.6], concerning large values of $p$. In that range the $n$-invariant least energy nodal solutions $U_{p, n}$ are nonradial for every $n=$ 
$1, \ldots\left\lceil\frac{2+\alpha}{2} \kappa-1\right\rceil$, where $\kappa \approx 5.1869$ is another fixed number related to the Morse index of $u_{p}^{*}$ for large values of $p$. Further they are distinct one from another thanks to a monotonicity result in [17. Since $\kappa>\beta+2$, we see that for $n=\left[\frac{2+\alpha}{2} \beta+1\right] \ldots\left\lceil\frac{2+\alpha}{2} \kappa-1\right\rceil$ the curve $p \mapsto U_{p, n}$ coincide with the one of radial least energy nodial solutions $p \mapsto u_{p}^{*}$ for $p$ under a certain value $p_{n}$, and then it bifurcates becoming nonradial. We conjecture that, specularly, the least energy solutions $U_{p, n}$ are nonradial (respectively, radial) for every values of $p>1$ when $n \leq\left\lceil\frac{2+\alpha}{2} \beta-1\right\rceil$ (respectively, $n \geq\left\lceil\frac{2+\alpha}{2} \kappa\right\rceil$ ).

\section{Preliminary Remarks on the Limit PROBlem}

The profile of solutions to the Hénon problem

$$
\begin{cases}-\Delta u=|x|^{\alpha}|u|^{p-1} u & \text { in } B \\ u=0 & \text { on } \partial B,\end{cases}
$$

is related to a weighted eigenvalue problem for the Laplacian, namely

1.3

$$
\begin{cases}-\Delta \omega=\mu|x|^{\alpha} \omega & \text { in } B, \\ \omega=0 & \text { on } \partial B .\end{cases}
$$

Indeed it is easy to prove the following general fact.

Lemma 2.1. Let $p_{n} \rightarrow 1$ and $u_{n}$ any nontrivial solution to (1.1) with $p$ replaced by $p_{n}$. If $\left\|u_{n}\right\|_{\infty}^{p_{n}-1} \leq C$, then there exists an eigenvalue $\mu$ of (1.3) with eigenfunction $\omega$ such that $\|\omega\|_{\infty}=1$ and (up to an extracted sequence)

$$
\left\|u_{n}\right\|_{\infty}^{p_{n}-1} \longrightarrow \mu \quad \text { and } \quad \frac{u_{n}}{\left\|u_{n}\right\|_{\infty}} \longrightarrow \omega \text { in } C^{2}(B) \text { and in } C(\bar{B})
$$

Proof. Certainly $\left\|u_{n}\right\|_{\infty}^{p_{n}-1}$ converges to a nonnegative number, say it $\mu$, up to an extracted sequence. Next $\bar{u}_{n}(x)=\frac{u_{n}(x)}{\left\|u_{n}\right\|_{\infty}}$ satisfies

$$
\begin{cases}-\Delta \bar{u}_{n}=|x|^{\alpha}\left\|u_{n}\right\|_{\infty}^{p_{n}-1}\left|\bar{u}_{n}\right|^{p_{n}-1} \bar{u}_{n} & \text { in } B, \\ \bar{u}_{n}=0 & \text { on } \partial B .\end{cases}
$$

and is nontrivial since $\left\|\bar{u}_{n}\right\|_{\infty}=1$. Hence $\left\|u_{n}\right\|_{\infty}^{p_{n}-1}$ cannot vanish (and so $\mu>0$ ) because by maximum principle

$$
\bar{u}_{n}=(-\Delta)^{-1}|x|^{\alpha}\left\|u_{n}\right\|_{\infty}^{p_{n}-1}\left|\bar{u}_{n}\right|^{p_{n}-1} \bar{u}_{n} \leq\left\|u_{n}\right\|_{\infty}^{p_{n}-1}(-\Delta)^{-1}(1) .
$$

Moreover

$$
\left(\left|\bar{u}_{n}\right|^{p_{n}-1}-1\right) \bar{u}_{n} \rightarrow 0 \quad \text { uniformly. }
$$

Indeed for any fixed $n$ we have $\left(\left|\bar{u}_{n}\right|^{p_{n}-1}-1\right) \bar{u}_{n}=0$ if $\bar{u}_{n}=0$, otherwise from the equality

$$
a^{s}-1=s \log a \int_{0}^{1} a^{t s} d t \quad \text { as } a>0, s \in \mathbb{R},
$$

we deduce that

$$
\begin{aligned}
\left|\left(\left|\bar{u}_{n}\right|^{p_{n}-1}-1\right) \bar{u}_{n}\right| & \leq\left.\left(p_{n}-1\right)|\log | \bar{u}_{n}\left|\int_{0}^{1}\right| \bar{u}_{n}\right|^{1+t\left(p_{n}-1\right)} d t \mid \\
& \leq c\left(p_{n}-1\right)\left|\bar{u}_{n}\right|^{1 / 2} \leq c\left(p_{n}-1\right) .
\end{aligned}
$$

So $\bar{u}_{n}$ converges weakly to a function $\omega$ that solves (1.3) for $\mu=\lim \left\|u_{n}\right\|_{\infty}^{p_{n}-1}$, and by ellipticity $\bar{u}_{n} \rightarrow \omega$ in $C^{2}(B)$ and uniformly on $\bar{B}$. From this it also follows that $\|\omega\|_{\infty}=1$, concluding the proof of (2.1).

It is not hard to obtain a better asymptotic description which shall be of use later on. 
Corollary 2.2. Let $p_{n} \rightarrow 1, u_{n}, \mu$ and $\omega$ as in the previous Lemma, and define

$$
c=\frac{-\int_{B}|x|^{\alpha} \log |\omega| \omega^{2} d x}{\int_{B}|x|^{\alpha} \omega^{2} d x} .
$$

Then as $n \rightarrow \infty$ we have

$$
\begin{aligned}
& \left\|u_{n}\right\|_{\infty}^{p_{n}-1}=\mu\left(1+c\left(p_{n}-1\right)\right)+o\left(p_{n}-1\right), \\
& \mu^{-\frac{1}{p_{n}-1}} u_{n} \longrightarrow e^{c} \omega \quad \text { in } C(\bar{B}) .
\end{aligned}
$$

This facts have been proved in [19] in some particular cases, but their proof still work in wide generality. We report it here for the reader convenience.

Proof. To obtain (2.6) we compute

$$
\left\|u_{n}\right\|_{\infty}^{p_{n}-1} \int_{B}|x|^{\alpha}\left|\bar{u}_{n}\right|^{p_{n}-1} \bar{u}_{n} \omega d x=\frac{1}{\left\|u_{n}\right\|_{\infty}} \int_{B}|x|^{\alpha}\left|u_{n}\right|^{p_{n}-1} u_{n} \omega d x=\frac{1}{\left\|u_{n}\right\|_{\infty}} \int_{B} \nabla u_{n} \nabla \omega d x
$$

because $u_{n}$ solves (1.1). Next using that $\omega$ solves (1.3) we end up with

Hence

$$
=\frac{\mu}{\left\|u_{n}\right\|_{\infty}} \int_{B}|x|^{\alpha} u_{n} \omega d x=\mu \int_{B}|x|^{\alpha} \bar{u}_{n} \omega d x
$$

$$
\begin{array}{r}
\left(\left\|u_{n}\right\|_{\infty}^{p_{n}-1}-\mu\right) \int_{B}|x|^{\alpha}\left|\bar{u}_{n}\right|^{p_{n}-1} \bar{u}_{n} \omega d x=\mu \int_{B}|x|^{\alpha}\left(1-\left|\bar{u}_{n}\right|^{p_{n}-1}\right) \bar{u}_{n} \omega d x \\
=-\mu\left(p_{n}-1\right) \int_{B}|x|^{\alpha} \log \left|\bar{u}_{n}\right| \int_{0}^{1}\left|\bar{u}_{n}\right|^{t\left(p_{n}-1\right)} d t \bar{u}_{n} \omega d x
\end{array}
$$

Summing up we have

$$
\frac{\left\|u_{n}\right\|_{\infty}^{p_{n}-1}-\mu}{\mu\left(p_{n}-1\right)}=\frac{-\int_{B}|x|^{\alpha} \log \left|\bar{u}_{n}\right| \int_{0}^{1}\left|\bar{u}_{n}\right|^{t\left(p_{n}-1\right)} d t \bar{u}_{n} \omega d x}{\int_{B}|x|^{\alpha}\left|\bar{u}_{n}\right|^{p_{n}-1} \bar{u}_{n} \omega d x} \rightarrow c
$$

as $n \rightarrow \infty$. Indeed the uniform convergence of $\bar{u}_{n}$ yields that

$$
\int_{B}|x|^{\alpha}\left|\bar{u}_{n}\right|^{p_{n}-1} u_{n} \omega d x \rightarrow \int_{B}|x|^{\alpha} \omega^{2} d x
$$

Further $\left.|\log | \bar{u}_{n}\left|\int_{0}^{1}\right| \bar{u}_{n}\right|^{t\left(p_{n}-1\right)} d t \bar{u}_{n}\left|\leq \sup _{s \in(0,1]}\right| s \log s \mid<\infty$ and so the Dominated Convergence Theorem yields

$$
\int_{B}|x|^{\alpha} \log \left|\bar{u}_{n}\right| \int_{0}^{1}\left|\bar{u}_{n}\right|^{t\left(p_{n}-1\right)} d t \bar{u}_{n} \omega d x \rightarrow \int_{B}|x|^{\alpha} \log |\omega| \omega^{2} d x .
$$

Eventually (2.7) follows because

$$
\mu^{-\frac{1}{p_{n}-1}} u_{n}=\left(\frac{\left\|u_{n}\right\|_{\infty}^{p_{n}-1}}{\mu}\right)^{\frac{1}{p_{n}-1}} \bar{u}_{n}=\left(1+c\left(p_{n}-1\right)+o\left(p_{n}-1\right)\right)^{\frac{1}{p_{n}-1}} \bar{u}_{n} \longrightarrow e^{c} \omega
$$

in $C(\bar{B})$ by (2.1).

Let us compute explicitly the eigenvalues and eigenfunctions of (1.3), which are related to the Bessel function of first kind

$$
\mathcal{J}_{\beta}(r)=r^{\beta} \sum_{k=0}^{+\infty} \frac{(-1)^{i} k}{k ! \Gamma(k+1+\beta)}\left(\frac{r}{2}\right)^{2 k}
$$

when $\beta=\frac{N-2+2 n}{2+\alpha}$ for some $n \in \mathbb{N}$. Here and henceforth we write

$$
z_{i}(\beta) \text { for the } i^{t h} \text { zero of } \mathcal{J}_{\beta} \text {, as } i \in \mathbb{N}, i \geq 1 \text {, }
$$


$\lambda_{n}=n(N-2+n)$ for the sequence of the eigenvalues of the Laplace Beltrami operator on $\mathbb{S}_{N-1}, N_{n}=\frac{(N+2 j-2)(N+n-3) !}{(N-2) ! n !}$ for its multiplicity, and $Y_{n, j}$ for the eigenfunctions of the Laplace Beltrami operator on $\mathbb{S}_{N-1}$, i.e. the spherical harmonics, as $n, j \in \mathbb{N}, j=1, \ldots N_{n}$.

Lemma 2.3. The eigenvalues of (1.3) are

$$
\mu_{n, i}=\left(\frac{2+\alpha}{2} z_{i}\left(\frac{N-2+2 n}{2+\alpha}\right)\right)^{2}
$$

and the related eigenfunctions are

$$
\omega_{n, i}(x)=|x|^{-\frac{N-2}{2}} \mathcal{J}_{\frac{N-2+2 n}{2+\alpha}}\left(z_{i}\left(\frac{N-2+2 n}{2+\alpha}\right)|x|^{\frac{2+\alpha}{2}}\right) Y_{n, j}\left(\frac{x}{|x|}\right) .
$$

Proof. Let $\omega \in H_{0}^{1}(B)$ solve the equation in (1.3). We decompose it along the spherical harmonics and write

$$
\omega(x)=\sum_{n=0}^{\infty} \sum_{j=1}^{N_{n}} \psi_{n, j}(|x|) Y_{n, j}\left(\frac{x}{|x|}\right) .
$$

An easy computation shows that $\psi_{n, j}$ are characterized by

$$
\begin{cases}-\left(r^{N-1} \psi^{\prime}\right)^{\prime}=r^{N-1}\left(r^{\alpha} \mu-\frac{\lambda_{n}}{r^{2}}\right) \psi & \text { for } 0<r<1, \\ \psi \in H_{0, \text { rad }}^{1}(B) & \text { and also } \\ \psi /|x| \in L^{2}(B) & \text { if } n \geq 1\end{cases}
$$

Next we perform the change of variable

$$
t=r^{\frac{2+\alpha}{2}}, \quad \phi(t)=\psi(r) .
$$

The function $\psi$ solves the equation in (2.13) if and only if

$$
t^{2} \phi^{\prime \prime}+\frac{2 N-2+\alpha}{2+\alpha} t \phi^{\prime}+\left(\frac{2}{2+\alpha}\right)^{2}\left(\mu t^{2}-\lambda_{n}\right) \phi=0 \quad \text { for } 0<t<1 .
$$

If $N=2$ (2.14) is a Bessel equation, otherwise is sufficient to perform a further transformation, namely

to obtain the Bessel equation

$$
\hat{\phi}(t)=t^{\frac{N-2}{2+\alpha}} \phi(t)
$$

$$
t^{2} \hat{\phi}^{\prime \prime}+t \hat{\phi}^{\prime}+\left(\left(\frac{2 \sqrt{\mu}}{2+\alpha}\right)^{2} t^{2}-\left(\frac{N-2+2 n}{2+\alpha}\right)^{2}\right) \hat{\omega}=0 .
$$

Here we have also used the explicit value $\lambda_{n}=n(N-2+n)$. The solutions of (2.15) are linear combinations of the Bessel functions of first and second kind, precisely

$$
\hat{\phi}(t)=C_{1} \mathcal{J}_{\frac{N-2+2 n}{2+\alpha}}\left(\frac{2 \sqrt{\mu} t}{2+\alpha}\right)+C_{2} \mathcal{Y}_{\frac{N-2+2 n}{2+\alpha}}\left(\frac{2 \sqrt{\mu} t}{2+\alpha}\right) .
$$

Coming back to $\psi(r)=r^{-\frac{N-2}{2}} \hat{\phi}\left(r^{\frac{2+\alpha}{2}}\right)$ and imposing that $\psi \in H_{0, \text { rad }}^{1}(B)$ one sees that the coefficient $C_{2}$ must be zero, and the condition $\psi(1)=0$ yields that $\frac{2 \sqrt{\mu}}{2+\alpha}$ is a zero of the

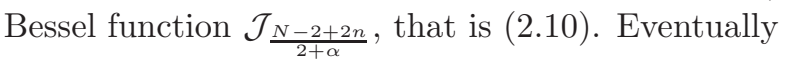

$$
\psi(r)=C r^{-\frac{N-2}{2}} \mathcal{J}_{\frac{N-2+2 n}{2+\alpha}}\left(z_{n, i} r^{\frac{2+\alpha}{2}}\right),
$$

and the decomposition (2.12) yields (2.11). 
Remark 2.4. The same arguments of the proof of Lemma 2.3 show that the radial eigenvalues of (1.3), i.e. the eigenvalues whose corresponding eigenfunctions belong to $H_{\mathrm{rad}}^{1}(B)$, are

$$
\mu_{0, i}=\left(\frac{2+\alpha}{2} z_{i}\left(\frac{N-2}{2+\alpha}\right)\right)^{2}
$$

Each of them has only one radial eigenfunction (up to a multiplicative constant) given by

$$
\omega_{0, i}(r)=r^{-\frac{N-2}{2}} \mathcal{J}_{\frac{N-2}{2+\alpha}}\left(z_{i}\left(\frac{N-2}{2+\alpha}\right) r^{\frac{2+\alpha}{2}}\right) .
$$

\section{RADiAL SOLUTIONS}

In this section we deal with radial solutions, for which a more detailed description of both the asymptotic profile and the Morse index can be given.

We consider the number of nodal zones $m$ as fixed, and write $u_{p}$ for the radial solution to (1.1) with $m$ nodal zones. It is unique up to the sign (see 25) and to fix idea we shall take that $u_{p}(0)>0$. We denote by $0<r_{1, p}<\ldots r_{m, p}=1$ the nodal radii of $u_{p}$, so that $u_{p}\left(r_{i, p}\right)=0$ as $i=1, \ldots m$. It is not hard to see by ODE techniques that $u_{p}$ has only one critical point in any nodal interval $A_{1}=\left[0, r_{1, p}\right)$ or $A_{i}=\left(r_{i-1, p}, r_{i, p}\right)$ if $i=2, \ldots m$, which shall be denoted by $s_{i-1, p}$ henceforth. Moreover $s_{0, p}=0$ is the global maximum point and $u_{p}(0)>-u_{p}\left(s_{1, p}\right)>u_{p}\left(s_{2, p}\right)>\ldots(-1)^{m-1} u_{p}\left(s_{m-1, p}\right)$. We refer to [5. Proposition 4.1] for a detailed proof.

Here we see that when $p$ approaches $1,\left\|u_{p}\right\|^{p-1}$ stays bounded, none of the nodal zones disappears and a suitable rescaling of $u_{p}$ converges to the $m^{t h}$ radial eigenfuntion of (1.3), which gives Theorem 1.1 .

Proof of Theorem 1.1. Let us check first that $\left\|u_{p}\right\|_{\infty}^{p-1}=\left|u_{p}(0)\right|^{p-1}$ is bounded for $p$ close to 1 . If not there exists a sequence $p_{n} \rightarrow 1$ such that

$$
\tau_{n}=\left\|u_{p_{n}}\right\|_{\infty}^{\frac{p_{n}-1}{2+\alpha}} \rightarrow \infty \quad \text { as } n \rightarrow+\infty
$$

so we look at the rescaled function

$$
U_{n}(x)=\frac{1}{\left\|u_{p_{n}}\right\|_{\infty}} u_{p_{n}}\left(\frac{x}{\tau_{n}}\right), \quad \text { as } x \in B_{\tau_{n}}=\left\{x \in \mathbb{R}^{N}:|x|<\tau_{n}\right\},
$$

that satisfies

$$
\begin{cases}-\Delta U_{n}=|x|^{\alpha}\left|U_{n}\right|^{p_{n}-1} U_{n}, & \text { in } B_{\tau_{n}} \\ \left|U_{n}\right| \leq U_{n}(0)=1, & \text { on } \partial B_{\tau_{n}} .\end{cases}
$$

Because $|x|^{\alpha}\left|U_{n}\right|$ is locally bounded, the function $U_{n}$ converges locally uniformly in $\mathbb{R}^{N}$ to a radial function $U$ which solves

$$
\left\{\begin{array}{l}
-\Delta U=|x|^{\alpha} U, \quad \text { in } \mathbb{R}^{N} \\
|U| \leq U(0)=1
\end{array}\right.
$$

Remark that the number of nodal zones of $U$ can not overpass the one of $U_{n}$, that is $m$. Indeed inside each nodal zone $U_{n}$ has fixed sign and converges uniformly to $U$. Therefore $U$ cannot change sign and Hopf Lemma yields that no further zero can appear.

On the other hand $w(r)=r^{\frac{N-2}{2+\alpha}} U\left(\left(\frac{2 r}{2+\alpha}\right)^{\frac{2}{2+\alpha}}\right)$ solves a Bessel equation

$$
r^{2} w^{\prime \prime}+r w^{\prime}+\left(r^{2}+\left(\frac{N-2}{2+\alpha}\right)^{2}\right) w=0
$$

and since it is bounded near at the origin $w(r)=A \mathcal{J}_{\frac{N-2}{2+\alpha}}(r)$, where $\mathcal{J}$ stands for the Bessel function of first kind. This is not possible because $w$ has an infinite number of nodal zones, 
proving that $\left\|u_{p}\right\|_{\infty}^{p-1} \leq C$.

Hence Lemma 2.1 ensures that when $p_{n} \rightarrow 1$ then the function $\bar{u}_{n}(x)=\frac{u_{n}(x)}{\left\|u_{n}\right\|_{\infty}}$ converges to an eigenfunction $\omega$ of (1.3) related to the eigenvalue $\mu=\lim \left\|u_{n}\right\|_{\infty}^{p_{n}-1}$. Of course $\omega$ has to be radial, it remains to show that it has exactly $m$ nodal zones. Actually the first nodal zone, say it $B_{r_{n}}=\left\{x:|x|<r_{n}\right\}$, can not collapse to a null set because multiplying the equation in (1.1) by $u_{n}$ and integrating on $B_{r_{n}}$ one sees that

$$
\begin{aligned}
\int_{B_{r_{n}}}\left|\nabla u_{n}\right|^{2} d x & =\int_{B_{r_{n}}}|x|^{\alpha}\left|u_{n}\right|^{p+1} d x \leq r_{n}^{\alpha}\left\|u_{n}\right\|_{\infty}^{p_{n}-1} \int_{B_{r_{n}}}\left|u_{n}\right|^{2} d x \\
& \leq C r_{n}^{N+\alpha}\left\|u_{n}\right\|_{\infty}^{p_{n}-1} \int_{B_{r_{n}}}\left|\nabla u_{n}\right|^{2} d x
\end{aligned}
$$

by the Poincaré inequality in the ball $B_{r_{n}}$. Remark that it also follows that $\left\|u_{n}\right\|_{\infty}^{p_{n}-1}$ does not vanish and in particular $\mu>0$.

The last nodal zone can not disappear either. To see this fact we denote by $s_{n}$ the last zero of $u_{n}, \tau_{n}=\left\|u_{n}\right\|_{\infty}^{\frac{p_{n}-1}{2+\alpha}}$ as before and rule out the occurrence $R_{n}:=\tau_{n}\left(1-s_{n}\right) \rightarrow 0$. To this aim we look at the rescaled sequence

$$
\zeta_{n}(r)=\frac{1}{\left\|u_{n}\right\|_{\infty}}\left|u_{n}\left(s_{n}+\frac{r}{\tau_{n}}\right)\right|, \quad \text { as } 0<r<R_{n}
$$

Now $0<\zeta_{n} \leq 1$ on $\left(0, R_{n}\right)$ and it solves

$$
\left\{\begin{array}{l}
-\left(\left(r+\tau_{n} s_{n}\right)^{N-1} \zeta_{n}^{\prime}\right)^{\prime}=\left(r+\tau_{n} s_{n}\right)^{N-1+\alpha} \zeta_{n}^{p} \quad 0<r<R_{n}, \\
\zeta(0)=\zeta\left(R_{n}\right)=0 .
\end{array}\right.
$$

So, recalling that we have already proved that $\tau_{n} s_{n} \geq \delta>0$, we compute

$$
\begin{aligned}
\int_{0}^{R_{n}}\left|\zeta_{n}^{\prime}\right|^{2} d r & \leq \frac{1}{\delta^{N-1}} \int_{0}^{R_{n}}\left(r+\tau_{n} s_{n}\right)^{N-1}\left|\zeta_{n}^{\prime}\right|^{2} d r=\frac{1}{\delta^{N-1}} \int_{0}^{R_{n}}\left(r+\tau_{n} s_{n}\right)^{N-1+\alpha} \zeta_{n}^{p_{n}+1} d r \\
& \leq \frac{\left(R_{n}+s_{n} \tau_{n}\right)^{N-1+\alpha}\left\|\zeta_{n}\right\|_{\infty}^{p_{n}-1}}{\delta^{N-1}} \int_{0}^{R_{n}} \zeta_{n}^{2} d r
\end{aligned}
$$

and using Wirtinger inequality and the boundedness of $R_{n}+s_{n} \tau_{n}$ and $\left\|\zeta_{n}\right\|_{\infty}$ we end up with

$$
\leq C R_{n}^{2} \int_{0}^{R_{n}}\left|\zeta_{n}^{\prime}\right|^{2} d r
$$

which forbids $R_{n} \rightarrow 0$. Similarly one can see that none of the other nodal zones can vanish, and so $\omega$ has at least $m$ nodal zones. But no more nodal zones can appear because inside each nodal zone $\omega$ is the uniform limit of $\bar{u}_{n}$ which has fixed sign.

Eventually $\mu$ is the $m^{\text {th }}$ radial eigenvalue for (1.3) and Remark 2.4 completes the proof of (1.4), (1.5), (1.6). The constant in (1.5) comes from the condition $\omega(0)=1$.

3.1. Computation of the Morse index. In this subsection we prove Theorem 1.2, by taking advantage of a characterization of the Morse index given in 4 . Let us recall that the Morse index of any solution $u_{p}$ to (1.1), that we denote by $m\left(u_{p}\right)$, is connected with the linearized operator

$$
L_{u_{p}} \psi:=-\Delta \psi-p|x|^{\alpha}\left|u_{p}\right|^{p-1} \psi
$$

and the quadratic form

$$
\mathcal{Q}_{u_{p}} \psi=\int_{B}|\nabla \psi|^{2}-p \int_{B}|x|^{\alpha}\left|u_{p}\right|^{p-1} \psi^{2},
$$


and can be defined as the number, counted with multiplicity, of the negative eigenvalues of

$$
\left\{\begin{array}{l}
L_{u_{p}} \psi=\Lambda_{k}(p) \psi \quad \text { in } B \\
\psi \in H_{0}^{1}(B)
\end{array}\right.
$$

or equivalently as the maximal dimension of a subspace of $H_{0}^{1}(B)$ where $\mathcal{Q}_{u_{p}}$ is negative defined. In [4, Proposition 1.1] an alternative definition of Morse index has been given by using a singular eigenvalue problem

$$
\left\{\begin{array}{l}
L_{u_{p}} \widehat{\psi}=\frac{\widehat{\Lambda}_{k}(p)}{|x|^{2}} \widehat{\psi} \quad \text { in } B \\
\widehat{\psi} \in \mathcal{H}_{0},
\end{array}\right.
$$

where $\mathcal{H}_{0}$ denotes the subspace of $H_{0}^{1}(B)$

$$
\mathcal{H}_{0}=\left\{\psi \in H_{0}^{1}(B):|x|^{-1} \psi \in L^{2}(B)\right\} .
$$

Precisely $m\left(u_{p}\right)$ is the number, counted with multiplicity, of the negative eigenvalues of (3.5), or equivalently as the maximal dimension of a subspace of $\mathcal{H}_{0}$ where $\mathcal{Q}_{u_{p}}$ is negative defined.

Hereafter we focus on the radial solution with exactly $m$ nodal zones, which shall be denoted by $u_{p}$, again. For such solution an even more effective description of its Morse index can be done by taking advantage from the transformation introduced in [18]

$$
t=r^{\frac{2+\alpha}{2}}, \quad w(t)=u(r),
$$

which maps the space $H_{0, \text { rad }}^{1}(B)$ into

$$
\begin{aligned}
H_{0, M}^{1}:=\{v:(0,1) \rightarrow \mathbb{R}: \quad & v \text { is measurable and has a first order weak derivative } v^{\prime} \\
& \text { with } \left.\int_{0}^{1} r^{M-1}\left(v^{2}+\left|v^{\prime}\right|^{2}\right) d r<+\infty\right\}
\end{aligned}
$$

for

$$
M=M(N, \alpha):=\frac{2(N+\alpha)}{2+\alpha} .
$$

As shown in [4, Proposition 4.9], $u_{p}$ is transformed by (3.6) into the unique (up to the $\operatorname{sign}$ ) solution of the "radially extended" Lane-Emden problem

$$
\left\{\begin{array}{l}
-\left(t^{M-1} w^{\prime}\right)^{\prime}=\left(\frac{2}{2+\alpha}\right)^{2} t^{M-1}|w|^{p-1} w, \quad 0<t<1 \\
w^{\prime}(0)=0, \quad w(1)=0
\end{array}\right.
$$

which has $m$ nodal zones and shall be denoted by $w_{p}$ hereafter.

In the same paper the computation of the Morse index of $u_{p}$ has been related to a singular Sturm-Liouville problem connected with $w_{p}$, namely

$$
\left\{\begin{array}{l}
-\left(t^{M-1} \phi_{i}^{\prime}\right)^{\prime}=t^{M-1}\left(W_{p}(t)+\frac{\nu_{i}(p)}{t^{2}}\right) \phi_{i} \quad \text { for } t \in(0,1), \\
\phi_{i} \in \mathcal{H}_{0, M}
\end{array}\right.
$$

where

$$
W_{p}(t)=p\left(\frac{2}{2+\alpha}\right)^{2}\left|w_{p}(t)\right|^{p-1}
$$

and $\mathcal{H}_{0, M}$ denotes the subspace of $H_{0, M}$ made up by functions which also satisfy

$$
\int_{0}^{1} t^{M-3} \phi^{2} d t<\infty
$$


It is useful to remark that the eigenvalues $\nu_{i}(p)$ are well defined only if $\nu_{i}(p)<\left(\frac{N-2}{2+\alpha}\right)^{2}$, and in this case they also have a variational characterization

$$
\begin{aligned}
& \nu_{1}(p)=\inf _{\phi \in \mathcal{H}_{0, M}} \operatorname{lop}_{w \neq 0}^{1} \frac{\int_{0}^{1} t^{M-1}\left(\left|\phi^{\prime}\right|^{2}-W_{p} \phi^{2}\right) d t}{\int_{0}^{1} t^{M-3} \phi^{2} d t}, \\
& \nu_{i}(p)=\inf _{\substack{\phi \in \mathcal{H}_{0, M} \\
w \perp_{M}\left\{\phi_{1}, \ldots, \phi_{i-1}\right\}}} \frac{\int_{0}^{1} t^{M-1}\left(\left|\phi^{\prime}\right|^{2}-W_{p} \phi^{2}\right) d t}{\int_{0}^{1} t^{M-3} \phi^{2} d t},
\end{aligned}
$$

see as shown in [4, Subsection 3.1]. Here the perpendicularity condition denoted by $\perp_{M}$ means

$$
\phi \perp_{M} \psi \Longleftrightarrow \int_{0}^{1} t^{M-3} \phi \psi d t=0 .
$$

Moreover by the analysis performed in [5, Section 4] the only negative eigenvalues of (3.10) are $\nu_{1}(p)<\nu_{2}(p)<\cdots<\nu_{m}(p)<0$ and they satisfy

$$
\nu_{i}(p)<-(M-1) \quad \text { as } i=1, \ldots m-1,
$$

for any value of the parameter $p$.

Putting together [4, Proposition 1.5] and [5, Theorem 1.3] gives

Proposition 3.1. Let $u_{p}$ be a radial solution to (1.1) with $m$ nodal zones. Then it is radially nondegenerate, its radial Morse index is $m$ and its Morse index is given by

$$
m\left(u_{p}\right)=\sum_{i=1}^{m} \sum_{j=0}^{\left\lceil J_{i}(p)-1\right\rceil} N_{j}
$$

where $J_{i}(p)=\frac{2+\alpha}{2}\left(\sqrt{\left(\frac{N-2}{2+\alpha}\right)^{2}-\nu_{i}(p)}-\frac{N-2}{2+\alpha}\right)$,

$\lceil s\rceil=\{\min n \in \mathbb{Z}: n \geq s\}$ denotes the ceiling function and

$N_{j}=\left\{\begin{array}{ll}1 & \text { when } j=0 \\ \frac{(N+2 j-2)(N+j-3) !}{(N-2) ! j !} & \text { when } j \geq 1\end{array}\right.$ is the multiplicity of the eigenvalue
$\lambda_{j}=j(N+j-2)$ for the Laplace-Beltrami operator in the sphere $\mathbb{S}_{N}$.

Furthermore the negative singular eigenvalues of (3.5) can be decomposed as

$$
\widehat{\Lambda}_{k}(p)=\left(\frac{2+\alpha}{2}\right)^{2} \nu_{i}(p)+\lambda_{j}
$$

as far as $\nu_{i}(p)<-\left(\frac{2}{2+\alpha}\right)^{2} \lambda_{j}$ for some $i=1, \ldots m$ and $j \geq 0$, while the related eigenfunctions are

$$
\widehat{\psi}_{k}(x)=\phi_{i}\left(|x|^{\frac{2+\alpha}{2}}\right) Y_{j}\left(\frac{x}{|x|}\right),
$$

where $\phi_{i}$ is an eigenfunction of (3.10) related to $\nu_{i}(p)$, and $Y_{j}$ is an eigenfunction for the Laplace-Beltrami operator in $\mathbb{S}_{N-1}$ related to $\lambda_{j}$.

In this way the asymptotic Morse index of $u_{p}$ can be computed by investigating the eigenvalues $\nu_{i}(p)$ of (3.10), which shall be the topic of the remaining of this subsection.

As a preliminary it is worth noticing that the convergence stated by Theorem 1.1 translates into the following one for $w_{p}$ and $W_{p}$, defined respectively in (3.6) and (3.11). 
Corollary 3.2. Let $z_{m}$ stand for $z_{m}\left(\frac{N-2}{2+\alpha}\right)$ according to (2.9). As $p \rightarrow 1$ we have

$$
\begin{aligned}
\left\|W_{p}\right\|_{\infty} & \rightarrow z_{m}^{2} \\
\frac{w_{p}(t)}{\left\|w_{p}\right\|_{\infty}} \rightarrow \Gamma\left(\frac{N+\alpha}{2+\alpha}\right) t^{-\frac{N-2}{2+\alpha}} \mathcal{J}_{\frac{N-2}{2+\alpha}}\left(z_{m} t\right) & \\
& =\Gamma\left(\frac{N+\alpha}{2+\alpha}\right) \sum_{k=0}^{+\infty} \frac{\left(-\frac{z_{m}^{2}}{4}\right)^{k}}{k ! \Gamma\left(k+\frac{N+\alpha}{2+\alpha}\right)} t^{2 k} \quad \text { in } C^{2}[0,1),
\end{aligned}
$$

and denoting by $0<t_{1}<\ldots t_{m}=1$ the zeros of $w_{p}$

$$
t_{i} \rightarrow \frac{z_{i}}{z_{m}} \quad \text { as } i=1, \ldots m-1 \text {. }
$$

Besides

$$
W_{p} \rightarrow z_{m}^{2} \quad \text { weakly in } L^{2}(0,1) \text {. }
$$

Proof. (3.19), (3.20) and (3.21) follow immediately by (1.4), (1.5) and (1.6). Further $W_{p}(t)=$ $\left\|W_{p}\right\|_{\infty}\left(\frac{w_{p}(t)}{\left\|w_{p}\right\|_{\infty}}\right)^{p-1}$ is bounded and converges uniformly to the constant $z_{m}^{2}$ on any closed interval contained in $[0,1) \backslash\left\{z_{1} / z_{m}, \ldots z_{m-1} / z_{m}\right\}$, so that the weak convergence follows trivially.

Also in the following when the parameter $\beta$ is omitted we mean $z_{i}=z_{i}\left(\frac{N-2}{2+\alpha}\right)$. Since the map $\beta \mapsto z_{i}(\beta)$ is continuous and increasing (see for instance [13), there exists

$$
\begin{aligned}
\beta_{i}=\beta_{i}(\alpha, N, m)>0 & \text { such that } z_{i}\left(\beta_{i}\right)\left(\text { the } i^{t h} \text { zero of the Bessel function } \mathcal{J}_{\beta_{i}}\right) \\
& \text { coincides with } z_{m}\left(\text { the } m^{t h} \text { zero of } \mathcal{J}_{\frac{N-2}{2+\alpha}}\right),
\end{aligned}
$$

moreover

$$
\beta_{1}>\ldots \beta_{m}=\frac{N-2}{2+\alpha} .
$$

The limit of the singular eigenvalues $\nu_{i}(p)$ can be expressed in terms of the parameters $\beta_{i}$ as follows.

Proposition 3.3. Consider the eigenvalue problem (3.10), with $W_{p}$ given as in (3.11), and $u_{p}$ is the radial solution to (1.1) with $m$ nodal zones. Then as $p \rightarrow 1$ we have

$$
\nu_{i}(p) \rightarrow\left(\frac{N-2}{2+\alpha}\right)^{2}-\beta_{i}^{2} \quad \text { as } i=1, \ldots m .
$$

In particular $\nu_{m}(p) \rightarrow 0$. Putting together Propositions 3.1 and 3.3 yields Theorem 1.2

Proof of Theorem 1.2. From the limit 3.24 one sees that the index $J_{i}(p)$ appearing in the Morse index formula (3.17) satisfies

$$
J_{i}(p) \rightarrow \frac{(2+\alpha) \beta_{i}-(N-2)}{2}
$$

as $p \rightarrow 1$. So when $\frac{(2+\alpha) \beta_{i}-(N-2)}{2}$ are not integer (1.7) follows, while when $\frac{(2+\alpha) \beta_{i}-(N-2)}{2}$ is integer for some $i$ we only get 1.8 .

Some preliminary lemmas are useful to prove Proposition 3.3. First we remark that all the eigenvalues of (3.10) are bounded from below in a neighborhood of $p=1$.

Lemma 3.4. There exists $C>0$ such that $\nu_{1}(p) \geq-C$ for $p$ close to 1 . 
Proof. By (3.19) $0 \leq W_{p}(t) \leq z_{m}^{2}+\varepsilon$ for $p$ sufficiently close to 1 . So for all $\psi \in \mathcal{H}_{0, M}$

$$
\int_{0}^{1} t^{M-1}\left(\left|\psi^{\prime}\right|^{2}-W_{p} \psi^{2}\right) d t \geq-\left(z_{m}^{2}+\varepsilon\right) \int_{0}^{1} t^{M-1} \psi^{2} d t \geq-\left(z_{m}^{2}+\varepsilon\right) \int_{0}^{1} t^{M-2} \psi^{2} d t,
$$

and the claim follows by the variational characterization (3.13).

Next we establish an ad-hoc Poincaré inequality. For $0 \leq a<b$ we denote by $H_{0, M}(a, b)$ the space of functions of $H^{1}(a, b)$ such that $\psi(b)=0$, endowed with the norm

$$
\left\|\psi ; H_{0, M}(a, b)\right\|=\int_{a}^{b} t^{M-1}\left|\psi^{\prime}\right|^{2} d t
$$

It is clear that $H_{0, M}(0,1)$ is the space $H_{0, M}$ already introduced. It is very easy to see that

Lemma 3.5. For every $\psi \in H_{0, M}(a, b)$ we have

$$
\int_{a}^{b} t^{M-1} \psi^{2} d t \leq \frac{b(b-a)}{M-1} \int_{a}^{b} t^{M-1}\left|\psi^{\prime}\right|^{2} d t
$$

Proof. Since $\psi$ has first derivative in $L^{2}$, it is continuous and differentiable a.e., and from $\psi(b)=0$ we get

$$
\psi(t)=\int_{t}^{b} \psi^{\prime}(r) d r
$$

Hence

$$
\begin{aligned}
\int_{a}^{b} t^{M-1} \psi^{2} d t & =\int_{a}^{b} t^{M-1}\left(\int_{t}^{b} \psi^{\prime}(s) d s\right)^{2} d t \\
& \leq \int_{a}^{b} t^{M-1}(b-t) \int_{t}^{b}\left|\psi^{\prime}(s)\right|^{2} d s d t \leq b(b-a) \int_{a}^{b} t^{M-2} \int_{t}^{b}\left|\psi^{\prime}(s)\right|^{2} d s d t \\
& =b(b-a) \int_{a}^{b}\left|\psi^{\prime}(s)\right|^{2} \int_{a}^{s} t^{M-2} d t d s \leq \frac{b(b-a)}{M-1} \int_{0}^{1} s^{M-1}\left|\psi^{\prime}(s)\right|^{2} d s
\end{aligned}
$$

Proof of Proposition 3.3. By (3.15), (3.16) and Lemma 3.4 for any sequence $p_{n} \rightarrow 1$ there is an extracted sequence (that we still denote by $p_{n}$ ) such that $\nu_{i}\left(p_{n}\right)$ converges to some $\bar{\nu}_{i}$. Moreover $\bar{\nu}_{i} \leq-(M-1)$ if $i=1, \ldots m-1$ and $-(M-1) \leq \bar{\nu}_{m} \leq 0$.

Let $\psi_{i, n} \in \mathcal{H}_{0, M}$ the eigenfunction related to $\nu_{i}\left(p_{n}\right)$ normalized so that $\left\|\psi_{i, n}\right\|_{\infty}=1$. We recall that by [4. Proposition 3.8 and Property 5 in Subsection 3.1] $\psi_{i, n} \in C[0,1] \cap C^{1}(0,1]$ has exactly $i$ nodal zones and for $t$ next to 0 and

$$
\left|\psi_{i, n}(t)\right| \leq C t^{\theta_{i, n}}, \quad\left|\psi_{i, n}^{\prime}(t)\right| \leq C t^{\theta_{i, n}-1},
$$

with $\theta_{i, n}=\sqrt{\left(\frac{M-2}{2}\right)^{2}-\nu_{i}\left(p_{n}\right)}-\frac{M-2}{2}$. It is worth remarking that the constants $C$ appearing here only depend by $\left\|\psi_{i, n}\right\|_{\infty}=1$, and therefore are general in the present situation. Further for $i=1, \ldots m-1$ the estimates in (3.26) assures that $\psi_{i, n}$ are equicontinuous on a set of type $[0, \varepsilon]$ because $\theta_{i, n} \geq 1$. This is not the case for $i=m$.

So the proof of (3.24) in the case $i=m$ will differ from the one for $i \leq m-1$.

First step: the first $m-1$ eigenvalues. We show that (3.24) holds as $i=1, \ldots m-1$, and in doing so we also see that

$$
\psi_{i, n}(t) \rightarrow A_{i} t^{-\frac{N-2}{2+\alpha}} \mathcal{J}_{\beta_{i}}\left(z_{m} t\right) \quad \text { uniformly in }[0,1]
$$

for some constant $A_{i} \neq 0$. 
Using $\psi_{i, n}$ as a test function in (3.10) gives

$$
\begin{aligned}
\int_{0}^{1} t^{M-1}\left|\psi_{i, p_{n}}^{\prime}\right|^{2} d t & =\int_{0}^{1} t^{M-1}\left(W_{p}+\frac{\nu_{i}\left(p_{n}\right)}{t^{2}}\right) \psi_{i, p_{n}}^{2} d t \\
& <\int_{0}^{1} t^{M-1} W_{p} \psi_{i, p_{n}}^{2} d t \leq C
\end{aligned}
$$

thanks to the normalization of $\psi_{i, n}$ and (3.19). Hence by the compact embedding of $H_{0, M}^{1}$ (see 4, Lemma 6.4]) $\psi_{i, n}$ converges to a function $\psi_{i}$ weakly in $H_{0, M}^{1}$, strongly in any $L_{M}^{q}$ for any $q>1$ (if $M=2=N$ ) or for any $1<q<\frac{2 M}{M-2}=\frac{2(N+\alpha)}{N-2}$ (if $M>2$, i.e. $N \geq 3$ ), and pointwise a.e. Moreover $\psi_{i, n} \rightarrow \psi_{i}$ also in uniformly on $[0,1]$ by Ascoli Theorem. Indeed we have already noticed that $\psi_{i, n}$ are equicontinuous on $[0, \varepsilon]$, while while for $t_{1}, t_{2} \in[\varepsilon, 1]$ we have

$$
\left|\psi_{i, n}\left(t_{1}\right)-\psi_{i, n}\left(t_{2}\right)\right| \leq \int_{t_{1}}^{t_{2}}\left|\psi_{i, n}^{\prime}(s)\right| d s \underset{\substack{\text { Holder } \\ \text { and [3.28) }}}{\leq} C\left(\int_{t_{1}}^{t_{2}} s^{1-M}\right)^{\frac{1}{2}} \leq C \varepsilon^{1-M} \sqrt{\left|t_{1}-t_{2}\right|} .
$$

Thanks to this and to the weak convergence in (3.22) one can pass to the limit into equation (3.10) and see that $\psi_{i}$ is a weak solution to

$$
-\left(t^{M-1} \psi_{i}^{\prime}\right)^{\prime}=t^{M-1}\left(z_{m}^{2}+\frac{\bar{\nu}_{i}}{t^{2}}\right) \psi_{i} \quad \text { as } 0<t<1 .
$$

The uniform convergence yields also that $\psi_{i}$ is not trivial (actually $\left\|\psi_{i}\right\|_{\infty}=1$ by the normalization) and has at most $i$ nodal zones. Let us check that it has exactly $i$ nodal zones, i.e. that none of the nodal zones of $\psi_{i, n}$ disappear. Let $a_{n}, b_{n}$ be two consecutive zeros of $\psi_{i, n}$, now the function $\psi_{i, n}$ restricted to the $\left(a_{n}, b_{n}\right)$ belongs to the space $H_{0, M}\left(a_{n}, b_{n}\right)$ introduced before Lemma 3.5, and clearly extending it to zero outside $\left(a_{n}, b_{n}\right)$ gives a function of $H_{0, M}$. Using this extension as a test function in (3.10) one sees that

$$
\begin{aligned}
\int_{a_{n}}^{b_{n}} t^{M-1}\left(\psi_{i, n}^{\prime}\right)^{2} d t & =\int_{a_{n}}^{b_{n}} t^{M-1}\left(W_{p}+\frac{\nu_{i}\left(p_{n}\right)}{t^{2}}\right) \psi_{i, n}^{2} d t \leq \int_{a_{n}}^{b_{n}} t^{M-1} W_{p} \psi_{i, n}^{2} d t \\
& \leq \int_{a_{n}}^{b_{n}} t^{M-1} \psi_{i, n}^{2} d t \leq \frac{C b_{n}\left(b_{n}-a_{n}\right)}{M-1} \int_{a_{n}}^{b_{n}} t^{M-1}\left(\psi_{i, n}^{\prime}\right)^{2} d t
\end{aligned}
$$

by the Poincaré inequality established in Lemma 3.5. If follows at once that neither $b_{n}$ or $b_{n}-a_{n}$ vanishes.

Next we define $\phi_{i}(t)=t^{\frac{N-2}{2+\alpha}} \psi_{i}\left(t / z_{m}\right)$. Starting from (3.29) it is easily seen that $\phi_{i}$ solves the Bessel equation

$$
t^{2} \phi_{i}^{\prime \prime}+t \phi_{i}^{\prime}+\left(t^{2}-\beta_{i}^{2}\right) \phi_{i}=0 \quad \text { for } \beta_{i}^{2}=z_{m}^{2}-\bar{\nu}_{i} .
$$

Since $\phi_{i}(0)=0$ we have that $\phi(t)=C \mathcal{J}_{\beta_{i}}(t)$, and as $\phi_{i}\left(z_{m}\right)=z_{m}^{\frac{N-2}{2+\alpha}} \psi_{i}(1)=0$ it follows that $z_{m}$ has to be a zero of the Bessel function $\beta_{i}$. Moreover we have seen that $\psi_{i}$ (and then also $\phi_{i}$ ) has $i$ nodal zones, so that $\beta_{i}$ is determined by the condition (3.23), which implies at once (3.24) and (3.27).

Second step: the last negative eigenvalue. It remains to check (3.24) for $i=m$, i.e. $\bar{\nu}_{m}=0$. To do this we compare the eigenfunction $\psi_{m, n}$ with $\bar{w}_{n}(t)=\frac{1}{\left\|w_{p_{n}}\right\|_{\infty}} w_{p_{n}}(t)$, that satisfies

$$
\left\{\begin{array}{l}
-\left(t^{M-1} \bar{w}_{n}^{\prime}\right)^{\prime}=\frac{1}{p_{n}} t^{M-1} W_{p_{n}} \bar{w}_{n} \quad \text { as } 0<t<1, \\
\bar{w}_{n}(0)=1, \bar{w}_{n}(1)=0 .
\end{array}\right.
$$

It is easy to establish the Picone type identity

$$
\left(t^{M-1}\left(\psi_{m, n}^{\prime} \bar{w}_{n}-\psi_{m, n} \bar{w}_{n}^{\prime}\right)\right)^{\prime}=t^{M-1}\left(\left(\frac{1}{p_{n}}-1\right) W_{p_{n}}(t)-\frac{\nu_{m}\left(p_{n}\right)}{t^{2}}\right) \psi_{m, n} \bar{w}_{n}
$$


as $0<t<1$. For a rigorous computation without requiring that $\psi_{m, n}$ is a classical solution we refer to [4, Lemma 3.13]. Thanks to (3.19) $\left(\frac{1}{p_{n}}-1\right) W_{p_{n}} \rightarrow 0$ uniformly, and assuming by contradiction that $\nu_{m}\left(p_{n}\right) \rightarrow \bar{\nu}<0$ it follows that

$$
\left(\frac{1}{p_{n}}-1\right) W_{p_{n}}-\frac{\nu_{m}\left(p_{n}\right)}{t^{2}}>0 \quad \text { as } 0<t<1
$$

for large $n$. Since both $\psi_{m, n}$ and $\bar{w}_{n}$ are null in $t=1$ and have exactly $m-1$ zeros on $(0,1)$, the Sturm-Picone's comparison Theorem yields that or $\psi_{m, n}$ is proportional to $\bar{w}_{n}$, or all the zeros of $\bar{w}_{n}$ follows the first zero of $\psi_{m, n}$, say it $\rho$. The first event is not possible because $\psi_{m, n}(0)=0$ by 3.26 while $\bar{w}_{n}(0)=1$. In the second case we may assume w.l.g. that $\bar{w}_{n}, \psi_{m, n}>0$ on $(0, \rho)$, so that $\psi_{m, n}^{\prime}(\rho)<0$ and $\bar{w}_{n}(\rho)>0$. Next integrating (3.31) between $t$ and $\rho$ and then letting $t \rightarrow 0$ gives

$$
\begin{array}{r}
\rho^{M-1} \psi_{m, n}^{\prime}(\rho) \bar{w}_{n}(\rho)-\lim _{t \rightarrow 0} t^{M-1}\left(\psi_{m, n}^{\prime} \bar{w}_{n}-\psi_{m, n} \bar{w}_{n}^{\prime}\right) \\
=\int_{0}^{\rho} t^{M-1}\left(\left(\frac{1}{p_{n}}-1\right) W_{p_{n}}-\frac{\nu_{m}\left(p_{n}\right)}{t^{2}}\right) \psi_{m, n} \bar{w}_{n} d t_{\text {3.32 }}^{>} 0 .
\end{array}
$$

But (3.26) guarantees that $\psi_{m, n}(t), t^{M-1} \psi_{m, n}^{\prime}(t) \rightarrow 0$ as $t \rightarrow 0$. Indeed also when $M=2$ we have $t^{M-1}\left|\psi_{m, n}^{\prime}(t)\right| \leq C t \sqrt{-\nu_{m}\left(p_{n}\right)}$ with $\nu_{m}\left(p_{n}\right)<0$. So we have reached the contradiction $\psi_{m, n}^{\prime}(\rho) \bar{w}_{n}(\rho)>0$, which concludes the proof.

Though the characterization of the Morse index in terms of the zeros of the Bessel function presented in (1.7) could not be completely satisfactory, because the laws $\beta \mapsto z_{i}(\beta)$ are not known explicitely, the position of $z_{i}(\beta)$ can be approximated by a numerical procedure, for instance by the command besselzero in MatLab. Combining this approximation with a dichotomy argument provides the approximated values of $\beta_{i}$ and therefore the Morse index of $u_{p}$. It is worth remarking that the computation of the asymptotic Morse index can be made more explicit in some particular cases.

Remark 3.6 (the planar case). In the plane the baseline Bessel function is $\mathcal{J}_{0}$, whose zeros are tabulated. Therefore approximated values of the parameters $\beta_{i}$ can be obtained in an elementary way. For instance in the case of two nodal zones we get $\beta_{1} \approx 2,305$, and formula (1.7) yields that for $p$ near at 1

$$
m\left(u_{p}\right)=2\left\lceil\frac{2+\alpha}{2} \beta\right\rceil, \quad \text { for } \beta \approx 2,305,
$$

if $\alpha \neq \alpha_{n}=2(n / \beta-1)$ (as $\left.n \geq 3\right)$, otherwise

$$
\left(2+\alpha_{n}\right) \beta \leq m\left(u_{p}\right) \leq\left(2+\alpha_{n}\right) \beta+2 .
$$

In particular the solution to the Lane-Emden equation $(\alpha=0)$ with two Nodal zones has Morse index 6, as already noticed in [19. For small positive values of $\alpha$ the Morse index remains 6 , while there is a critical value $\approx 0,6030$ above which the asymptotic Morse index increases to 8. This fact suggests that the set of the solutions to (1.1) changes in correspondence of that value of $\alpha$. We shall come back on this topic in next section.

In higher dimension the approximation of the parameters $\beta_{i}$ appearing in the computation of the Morse index can be numerically performed after having chosen a specific value for $\alpha$, which fixes the baseline Bessel function $\mathcal{J}_{\frac{N-2}{2+\alpha}}$. There is numerical evidence that

$$
z_{i}(\beta+2(m-i))<z_{m}(\beta)<z_{i}(\beta+2(m-i)+1) .
$$

Such estimate is not sufficient to single the exact Morse index out, except that in the Lane Emden case, for which one can infer that $\lim _{p \rightarrow 1} J_{i}^{m}(p) \in(2(m-i), 2(m-i)+1)$ and then, 
eventually

$$
m\left(u_{p}\right)=m+\sum_{i=1}^{m-1}(m-i)\left(N_{2 i-1}+N_{2 i}\right) \quad \text { for } p \text { close to } 1 .
$$

Let us remark that in dimension 2 (3.33) generalizes the computation in [19] (concerning the case of two nodal zones) as

$$
m\left(u_{p}\right)=m(2 m-1) .
$$

\section{4. $n$-INVARIANT SOLUTIONS}

We focus here on dimension $N=2$ with the aim of producing nonradial solutions for the almost-linear Henon problem (i.e. for $p$ close to 1). This is possible only in the framework of nodal solutions by the uniqueness result in [3, Theorem 3.1], which can be easily extended to the planar case. Let us remark by now that the Hénon problem is invariant for rotations around the origin, therefore any nonradial solution $u_{p}$ generates a family of nonradial solutions (the ones obtained by rotating $u_{p}$ of any given angle), and any claim about nonradial solutions can be stated "up to rotation".

We denote by $\mathcal{E}_{p}$ the energy functional associated to (1.1) i.e.

$$
\mathcal{E}_{p}(u):=\frac{1}{2} \int_{B}|\nabla u|^{2}-\frac{1}{p+1} \int_{B}|x|^{\alpha}|u|^{p+1},
$$

by $\mathcal{E}_{p}^{\prime}(u)$ for its Fréchet derivative computed at $u$, i.e.

$$
\mathcal{E}_{p}^{\prime}(u) \cdot v=\int_{B} \nabla u \nabla v d x-\int_{B}|x|^{\alpha}|u|^{p-1} u v d x
$$

and we introduce the Nehari set and the nodal Nehari set as

$$
\begin{aligned}
\mathcal{N}_{p} & :=\left\{v \in H_{0}^{1}(B): v \neq 0, \mathcal{E}_{p}^{\prime}(v) \cdot v=0\right\}, \\
\mathcal{N}_{p}^{\text {nod }} & :=\left\{v \in H_{0}^{1}: v^{ \pm} \neq 0, \mathcal{E}_{p}^{\prime}(v) \cdot v^{ \pm}=0\right\} .
\end{aligned}
$$

Here $v^{+}$and $v^{-}$stand for the positive and the negative part of $v$ respectively.

Due to the compact embedding of $H_{0}^{1}(B)$ in $L^{p}(B)$ for every $p>1, \min _{u \in \mathcal{N}_{p}^{\text {nod }}} \mathcal{E}_{p}(u)$ is attained at a nontrivial function, which is a weak and also classical solution to (1.1), and is known as the least energy nodal solution since it changes sign by construction. The existence of such least energy nodal solutions, together with some general properties, have been established in 8 and 9 . Let us recall the ones which shall turn useful to the present purpose.

Proposition 4.1. Let $U_{p}$ be a least energy nodal solution to (1.1). Then $U_{p}$ has exactly two nodal zones and its Morse index is 2.

Further, up to rotation, $U_{p}$ is symmetric w.r.t. the $x$ axis (i.e. $U_{p}(x,-y)=U_{p}(x, y)$ ) and nonincreasing w.r.t. the polar variable in the semicircle $B \cap\{(x, y): y>0\}$.

Of course one can repeat the same arguments on the subset of $H_{0}^{1}(B)$ made up by radial functions, thus ending with a least energy nodal radial solution which has exactly two nodal zones and radial Morse index equal to 2. By the uniqueness result in [25, we infer that the least energy nodal radial solution is nothing else that the radial solution with 2 nodal zones, to which we we will refer as $u_{p}^{*}$ in the following. Next, since in [5] it has been proved that the Morse index of any nodal radial solution is greater than 4 , it follows that $U_{p} \neq u_{p}^{*}$ for every $p$.

With the aim of producing other nonradial solutions, we introduce the so called $n$ invariant functions, studied in 19 in the Lane-Emden case, and also in 7] in the Hénon case (for large values of $p$ ). Precisely we denote by $H_{0, n}^{1}$ and $\mathcal{N}_{p, n}^{\text {nod }}$ the subsets of $H_{0}^{1}(B)$ and $\mathcal{N}_{p}^{\text {nod }}$ made up by that functions which are invariant for reflection across the horizontal axis 
(i.e. even w.r.t. $y$ ) and for rotations of an angle $2 \pi / n$. They can be easily described by using the polar coordinates $(r, \theta) \in[0, \infty) \times[-\pi, \pi]$ defined by the relation $(x, y)=(r \cos \theta, r \sin \theta)$, by means of

$$
\begin{aligned}
H_{0, n}^{1} & :=\left\{u \in H_{0}^{1}(B): u(r, \theta) \text { is even and } 2 \pi / n \text { periodic w.r.t. } \theta \text {, for every } r \in(0,1)\right\}, \\
\mathcal{N}_{p, n}^{\text {nod }} & :=\mathcal{N}_{p}^{\text {nod }} \cap H_{0, n}^{1} .
\end{aligned}
$$

Since of course also $H_{0, n}^{1}$ is compactly embedded in $L^{p}(B)$ for every $p>1$, for every integer $n \mathcal{E}_{p}(u)$ attains its minimum on $\mathcal{N}_{p, n}^{\text {nod }}$ at a nontrivial function $U_{p, n}$, which is a weak and also classical solution to (1.1), and we call least energy nodal n-symmetric solution. By Proposition 4.1 the least energy nodal solution belongs to $H_{0,1}^{1}$ and so it coincides with $U_{p, 1}$. In particular $U_{p, 1}$ is nonradial.

To understand whether $U_{p, n}$ is radial or not when $n \geq 2$, we make use of the $n$-Morse index, i.e. the maximal dimension of a subspace of $H_{0, n}^{1}$ in which the quadratic form $\mathcal{Q}_{u}$ (3.3) is negative defined, or equivalently, the number of negative eigenvalues of the linearized operator $L_{u}$, according to (3.4), which have corresponding eigenfunction in $H_{0, n}^{1}$. We refer hereafter to $m_{n}(u)$ as the $n$-symmetric Morse index of a solution $u$.

Repeating the arguments of [8] in the $n$-invariant functional space (see also [7]) one can see that

Lemma 4.2. The $n$-Morse index $U_{p, n}$ is equal to 2 for every integer $n$ and $p>1$.

For what concerns the least energy nodal radial solution $u_{p}^{*}$, its $n$ Morse index can be computed starting from the singular eigenvalues of (3.10) thanks to the analysis performed in [4].

Lemma 4.3. For every positive integer $n$ and $p>1$

$$
m_{n}\left(u_{p}^{*}\right)=2+\sum_{i=1}^{2}\left[\frac{1}{n}\left\lceil\frac{2+\alpha}{2} \sqrt{-\nu_{i}(p)}-1\right\rceil\right] .
$$

Here $\nu_{1}(p)$ and $\nu_{2}(p)$ are the only negative eigenvalues of (3.10) related to $W_{p}^{*}(t)=p\left(\frac{2+\alpha}{2}\right)^{2}\left|u_{p}^{*}\left(t^{\frac{2}{2+\alpha}}\right)\right|^{p-1}$.

Proof. [4, Corollary 4.11] yields that the $n$-Morse index of a radial solution is

$$
m_{n}\left(u_{p}^{*}\right)=\sum_{i=1}^{2} \sum_{j=0}^{\left\lceil\frac{2+\alpha}{2} \sqrt{-\nu_{i}(p)}-1\right\rceil} N_{j}^{n}
$$

where $N_{j}^{n}$ stands for the number of linearly independent eigenfunctions related to the $j^{\text {th }}$ eigenvalue of the Laplace Beltrami operator on the sphere $\mathbb{S}_{1}$ which are $n$-invariant. Recalling that the Laplace Beltrami eigenfunctions are linear combinations of $\cos j \theta$ and $\sin j \theta$, one sees that $N_{j}^{n}=1$ if $j=0$ or is a multiple of $n$ (since in that case $\cos j \theta$ is $n$-invariant), and zero otherwise. Hence for $i=1,2$ fixed, we have 1 eigenfunction related to $j=0$ (by (3.15), (3.16) ), and then another eigenfunction for every index $j=h n$ where $h$ is an integer such that $1 \leq h \leq \frac{1}{n}\left\lceil\frac{2+\alpha}{2} \sqrt{-\nu_{i}(p)}-1\right\rceil$, which totally gives $1+\left\lceil\frac{1}{n}\left\lceil\frac{2+\alpha}{2} \sqrt{-\nu_{i}(p)}-1\right\rceil\right]$ independent eigenfunctions in $H_{0, n}$ and concludes the proof.

Lemmas 4.2 and 4.3. together with the knowledge of the asymptotic Morse index of $u_{p}^{*}$ stated by Theorem 1.2, yield that some of the least-energy $n$-symmetric nodal solutions are nonradial for $p$ close to 1 .

Corollary 4.4. There exists $\bar{p}=\bar{p}(\alpha)>1$ such that $U_{p, n}$ are nonradial when $p \in(1, \bar{p})$ and $n=1, \ldots\left\lceil\frac{2+\alpha}{2} \beta-1\right\rceil$, where $\beta \approx 2,305$ is the fixed number mentioned in Remark 3.6 . 
Proof. Since $U_{p, n}$ is a least energy nodal solution and $H_{0, \mathrm{rad}}^{1} \subset H_{0, n}^{1}$, if it is radial it coincides with $u_{p}^{*}$, up to the sign. So by Lemma $4.2 U_{p, n}$ is nonradial whenever $m_{n}\left(u_{p}^{*}\right)>2$, which in turn, by Lemma 4.3. holds true provided that $n \leq\left\lceil\frac{2+\alpha}{2} \sqrt{-\nu_{1}(p)}-1\right\rceil$, or equivalently $n<\frac{2+\alpha}{2} \sqrt{-\nu_{1}(p)}$. Eventually the claim follows since $\frac{2+\alpha}{2} \sqrt{-\nu_{1}(p)} \rightarrow \frac{2+\alpha}{2} \beta$ by (3.24).

Nothing assures so far that this construction really produces $\left\lceil\frac{2+\alpha}{2} \beta-1\right\rceil$ distinct nonradial nodal solutions to (1.1): in principle $U_{p, n}$ could coincide. Further nothing has been said about the other $n$-invariant solutions with larger $n$. We find an answer to these questions by inspecting the asymptotic profile of $U_{p, n}$ when $p \rightarrow 1$. This issue has, in the author's opinion, interest for itself and will be the subject of next subsection.

4.1. Asymptotic profile of $n$-symmetric solutions. Here we describe the profile of $U_{p, n}$ when $p \rightarrow 1$. First we obtain a bound for $\left\|U_{p, n}\right\|_{\infty}^{p-1}$ which ensures, via Lemma 2.1, that $U_{p, n} /\left\|U_{p, n}\right\|_{\infty}$ converges to an eigenfunction of (1.3). Next we see that these limit eigenfunctions are nonradial and distinct one from another for $n=1, \ldots\left\lceil\frac{2+\alpha}{2} \beta-1\right\rceil$, while for the subsequent values of $n$ they coincide with the second radial eigenfunction.

Obtaining a bound for $\|u\|_{\infty}^{p-1}$ when $u$ is a $n$-invariant solution is far harder than in the radial setting, because it is not known a-priori where the extremal points accumulate when $p \rightarrow 1$. We show that a sufficient condition is that the $n$-Morse index is bounded from above. Such condition is certainly satisfied by the least energy $n$-invariant solutions thanks to Lemma 4.2 .

To this aim we focus on the circular sector

$$
S_{n}=\{(r, \theta): 0<r<1,0<\theta<\pi / n\},
$$

and introduce some notations and preliminary materials. The boundary of $S_{n}$ decomposes as $\partial S_{n}=\{O, A, B\} \cup \Gamma_{1} \cup \Gamma_{2} \cup \Gamma_{3}$ where $O$ is the origin, and in standard coordinates $A=\left(\cos \frac{\pi}{n}, \sin \frac{\pi}{n}\right), B=(1,0)$ and

$$
\begin{gathered}
\Gamma_{1}=\left\{(x, y): x^{2}+y^{2}=1, \cos \frac{\pi}{n}<x<1,0<y<\sin \frac{\pi}{n}\right\}, \\
\Gamma_{2}=\{(x, y): 0<x<1, y=0\}, \quad \Gamma_{3}=\left\{(x, y): \frac{y}{x}=\tan \frac{\pi}{n}, 0<x<\cos \frac{\pi}{n}\right\} .
\end{gathered}
$$

Of course when $n=2$ we mean $\Gamma_{3}=\{(x, y): x=0,0<y<1\}$. Next we set

$$
\begin{aligned}
& \widetilde{S}_{n}=\bar{S}_{n} \cap B=S_{n} \cup \Gamma_{2} \cup \Gamma_{3} \cup\{O\}, \\
& \mathcal{C}_{n}=\left\{v \in C\left(\bar{S}_{n}\right) \cap C^{1}\left(\widetilde{S}_{n}\right): v=0 \text { on } \Gamma_{1}, \partial_{\nu} v=0 \text { on } \Gamma_{2} \cup \Gamma_{3}\right\},
\end{aligned}
$$

where $\nu$ stands for the outer normal vector to the boundary of $S_{n}$. Now the restriction to $S_{n}$ of any function in $H_{0, n}^{1} \cap C^{1}(B)$ belongs to $\mathcal{C}_{n}$, and viceversa any function in $\mathcal{C}_{n}$ can be extended (by symmetry and periodicity) to a function in $H_{0, n}^{1} \cap C^{1}(B)$. In particular $u_{p} \in H_{0, n}^{1}$ is a classical solution of (1.1) if and only if its restriction to $S_{n}$ solves the mixed boundary problem

$$
\begin{cases}-\Delta u=|x|^{\alpha}|u|^{p-1} u & \text { in } S_{n}, \\ u=0 & \text { on } \Gamma_{1}, \\ \partial_{\nu} u=0 & \text { on } \Gamma_{2} \cup \Gamma_{3},\end{cases}
$$

and it is elementary to check that

Lemma 4.5. Let $u \in H_{0, n}^{1}$ be a solution of (1.1) whose $n$-Morse index is $m$. Then the eigenvalue problem

$$
\begin{cases}-\Delta w=\left(p|x|^{\alpha}|u|^{p-1}+\mu\right) w & \text { in } S_{n}, \\ w=0 & \text { on } \Gamma_{1}, \\ \partial_{\nu} w=0 & \text { on } \Gamma_{2} \cup \Gamma_{3} .\end{cases}
$$


has exactly $m$ negative eigenvalues. Equivalently, the maximal dimension of a subspace of $\mathcal{C}_{n}$ where the quadratic form

$$
\mathcal{Q}_{u}(w)=\int_{S_{n}}\left(|\nabla w|^{2}-p|x|^{\alpha}|u|^{p-1} w^{2}\right) d x
$$

is negative defined is exactly $m$.

The bound for $\left\|u_{p}\right\|_{\infty}^{p-1}$ is obtained by a refined blow-up argument which starts from (4.2) and ends up contradicting Lemma4.5. The blow-up procedure can bring to different domains for the limit problem, specifically $\mathbb{R}^{2}$ or an half space, or an angle. In this perspective we point out that the limit eigenvalue problems have infinite Morse index in the following sense.

Lemma 4.6. Let $\alpha \geq 0$ and $\Sigma \subset \mathbb{R}^{2}$ (to be specified later). We consider the quadratic form

$$
Q_{\Sigma}^{\alpha}(\phi)=\int_{\Sigma}\left(|\nabla \phi|^{2}-|x|^{\alpha} \phi^{2}\right) d x
$$

Then for every integer $k$ there exist $R>0$ and $k$ linearly independent functions with support contained in $\bar{B}_{R}$ which are zero on $\partial B_{R} \cap \Sigma$, are continuous on the closure of $\partial B_{R} \cap \Sigma$ and $C^{1}$ in its interior, satisfy

$$
Q_{\Sigma}^{\alpha}\left(\phi_{j}\right)<0 \quad \text { as } j=1, \ldots k
$$

and

(1) if $\Sigma=\mathbb{R}^{2}$, then $\phi_{j} \in H_{0, n}^{1}$.

(2) If $\Sigma$ is an half-plane of type $\{Q: Q \cdot P<0\}$, then $\phi_{j}$ are symmetric with respect to the direction orthogonal to $P$ and there exists $\delta>0$ such that $\phi_{j}=0$ in a strip $\{Q: Q \cdot P \geq-\delta\}$.

Proof. We use the notations of Lemma 2.3 and define, in polar coordinates

$$
\phi_{j}(r, \theta)=\mathcal{J}_{\frac{2 j n}{2+\alpha}}\left(\frac{Z_{j}}{R} r\right) \cos (j n \theta) \quad \text { in } B_{R}
$$

for $Z_{j}=z_{1}\left(\frac{2 j n}{2+\alpha}\right)$. They satisfy $-\Delta \phi_{j}=|x|^{\alpha}\left(\frac{Z_{j}}{R}\right)^{2} \phi_{j}$ pointwise on $B_{R}$ and vanish at $\partial B_{R}$. Extending them to zero outside $B_{R}$, multiplying the equation by $\phi_{j}$ and integrating by parts on $B_{R}$ (taking advantage from the boundary condition on $\partial B_{R}$ ) gives

$$
Q_{\mathbb{R}^{2}}^{\alpha}\left(\phi_{j}\right)=\int_{B_{R}}\left(\left|\nabla \phi_{j}\right|^{2}-|x|^{\alpha} \phi_{j}^{2}\right) d x=\left(\left(\frac{Z_{j}}{R}\right)^{2}-1\right) \int_{B_{R}}|x|^{\alpha} \phi_{j}^{2} d x<0
$$

for $j=1, \ldots k$ provided that $R>Z_{k}$. So we have obtained the functions requested by item (1).

Concerning the following item, since the quadratic form is invariant by rotation it is sufficient to make the proof only in one particular set of type (2) so we fix $P=(1,0)$ and $\Sigma=\{(x, y)$ : $x<0\}$. Next we take a cut-off function $\xi \in C^{1}(-\infty, 0]$ so that

$$
0 \leq \xi \leq 1, \quad \xi(t)=\left\{\begin{array}{ll}
1 & \text { as } t \leq-2 \delta, \\
0 & \text { as }-\delta \leq t \leq 0,
\end{array} \quad-2 / \delta \leq \xi^{\prime}(t) \leq 0 \quad \text { as }-2 \delta \leq t \leq-\delta\right.
$$

and define (in standard coordinates)

$$
\psi_{j}(x, y)=\phi_{j}(x, y) \xi(x) \quad \text { in } B_{R} \cap\{(x, y): x<0\} .
$$

It is clear that $\psi_{j}$ is zero on $\partial B_{R}$ and when $-\delta \leq x$, and even w.r.t. y. Besides $Q_{\Sigma}^{\alpha}\left(\psi_{j}\right) \rightarrow$ $Q_{\Sigma}^{\alpha}\left(\phi_{j}\right)=\frac{1}{2} Q_{\mathbb{R}^{2}}^{\alpha}\left(\phi_{j}\right)<0$ as $\delta \rightarrow 0$, concluding the proof of item (2). Indeed it is clear that 
$\int_{\Sigma}|x|^{\alpha}\left(\phi_{j} \xi\right)^{2} d x \rightarrow \int_{\Sigma}|x|^{\alpha} \phi_{j}^{2} d x$ as $\delta \rightarrow 0$, moreover since $\phi_{j}=0$ at $x=0$, using its $C^{1}$ regularity and the properties of $\xi$ gives

$$
\begin{array}{r}
\left|\int_{\Sigma}\left(\left|\nabla\left(\phi_{j} \xi\right)\right|^{2}-\left|\nabla \phi_{j}\right|^{2}\right) d x\right| \leq \int_{\Sigma \cap B_{R}}\left|\nabla \phi_{j}\right|^{2}\left(1-\xi^{2}\right) d x \\
+\int_{\Sigma \cap B_{R}} \phi_{j}^{2}\left|\xi^{\prime}\right|^{2} d x+2 \int_{\Sigma \cap B_{R}}\left|\phi_{j}\right|\left|\nabla \phi_{j}\right||\xi|\left|\xi^{\prime}\right| d x \leq C \text { meas }\left(B_{R} \cap\{-\delta \leq x \leq 0\}\right) .
\end{array}
$$

Remark 4.7. For $\alpha=0$ the quadratic form in $Q_{\Sigma}^{0}$ is invariant also for translation, so Lemma 4.6 continue to hold also for shifted sets of type $\Sigma+P_{0}$, for every $P_{0} \in \mathbb{R}^{2}$.

We are now in the position to prove the $L^{\infty}$ estimate.

Proposition 4.8. Let $p_{k} \rightarrow 1$ and $u_{k} \in H_{0, n}^{1}$ a sequence of solutions of (1.1) with $p=p_{k}$. If $m_{n}\left(u_{k}\right) \leq m<\infty$, then there exists a constant $C$ such that $\left\|u_{k}\right\|_{\infty}^{p_{k}-1} \leq C$ for large $k$.

Proof. We argue by contradiction and take that $\left\|u_{k}\right\|_{\infty}^{p_{k}-1} \rightarrow \infty$ along a subsequence, that we still denote by $p_{k}$. By the symmetry of $u_{k}$ there is $P_{k} \in S_{n}$ where $u_{k}$ achieves its extremal value, that we can take to be a maximum w.l.o.g. Up to another sequence $P_{k} \rightarrow \bar{P} \in \bar{S}_{n}$, and we argue differently according to the location of $\bar{P}$.

Before going on, let us introduce some notations and make some general considerations. We write $X=(x, y)$ for a generic point in $\mathbb{R}^{2}, P_{k}=\left(x_{k}, y_{k}\right), \bar{P}=(\bar{x}, \bar{y})$. Whenever $\bar{P} \neq 0$ we take as scaling parameter

$$
M_{k}=\left\|u_{k}\right\|_{\infty^{\frac{p_{k}-1}{2}}}^{{ }^{2}}|\bar{P}|^{\frac{\alpha}{2}},
$$

and introduce the change of variables

$$
X^{\prime}=P_{k}+\frac{X}{M_{k}}, \quad \widetilde{u}_{k}(X)=\frac{u_{k}\left(X^{\prime}\right)}{\left\|u_{k}\right\|_{\infty}} \text { as } X \in \Sigma_{k}=\left\{X \in \mathbb{R}^{2}: X^{\prime} \in S_{n}\right\} .
$$

The regular part of the boundary of $\Sigma_{k}$ is made up by the curves

$$
\begin{aligned}
\Gamma_{1, k}=\left\{X=(x, y):\left|X / M_{k}+P_{k}\right|=1,0<\frac{y+y_{k} M_{k}}{x+x_{k} M_{k}}<\tan \frac{\pi}{n}\right\} \\
\Gamma_{2, k}=\left\{X=(x, y):-M_{k} x_{k}<x<M_{k}\left(1-x_{k}\right), y=-M_{k} y_{k}\right\} \\
\Gamma_{3, k}=\left\{X=(x, y): \frac{y+y_{k} M_{k}}{x+x_{k} M_{k}}=\tan \frac{\pi}{n},-M_{k} x_{k}<x<M_{k}\left(\cos \frac{\pi}{n}-x_{k}\right),\right. \\
\left.-M_{k} y_{k}<y<M_{k}\left(\sin \frac{\pi}{n}-y_{k}\right)\right\},
\end{aligned}
$$

if $n \neq 2$, otherwise

$$
\Gamma_{3, k}=\left\{X=(x, y): x=-M_{k} x_{k},-M_{k} y_{k}<y<M_{k}\left(1-y_{k}\right)\right\} .
$$

Next $\widetilde{u}_{k}$ solves

$$
\begin{cases}-\Delta \widetilde{u}_{k}=\rho_{k}\left|\widetilde{u}_{k}\right|^{p_{k}-1} \widetilde{u}_{k} & \text { in } \Sigma_{k}, \\ \widetilde{u}_{k}=0 & \text { on } \Gamma_{1, k}, \\ \partial_{\nu} \widetilde{u}_{k}=0 & \text { on } \Gamma_{2, k} \cup \Gamma_{3, k}\end{cases}
$$

for

$$
\rho_{k}(X)=\frac{\left|X^{\prime}\right|^{\alpha}}{|\bar{P}|^{\alpha}}=\frac{\left|P_{k}+X / M_{k}\right|^{\alpha}}{|\bar{P}|^{\alpha}} .
$$

Notice that $M_{k} \rightarrow \infty$ and $\rho_{k} \rightarrow 1$ locally uniformly unless $\bar{P}=O$.

Further $\widetilde{u}_{k}(O)=1=\left\|\widetilde{u}_{k}\right\|_{\infty}$ for every $k$ and by standard elliptic estimates if $\Sigma$ is any open 
subset of $\mathbb{R}^{2}$ such that $\Sigma \subset \Sigma_{k}$ for large $k$, then $\widetilde{u}_{k}$ converges weakly in $H_{\text {loc }}^{1}(\Sigma)$ and in $C_{\text {loc }}(\Sigma)$ to a function $\widetilde{u}$ which solves

$$
-\Delta \widetilde{u}=\widetilde{u} \quad \text { in } \Sigma
$$

in weak sense (and therefore also in classical sense). Indeed for every $\varphi \in C_{0}^{\infty}(\Sigma)$, taken $k$ so large that the support of $\varphi$ in contained in $\Sigma_{k}$, we have

$$
\begin{aligned}
0 & =\int_{\Sigma}\left(\nabla \widetilde{u}_{k} \nabla \varphi-\rho_{k}\left|\widetilde{u}_{k}\right|^{p_{k}-1} \widetilde{u}_{k} \varphi\right) d X \\
& =\int_{\Sigma}\left(\nabla \widetilde{u}_{k} \nabla \varphi-\widetilde{u}_{k} \varphi\right) d X+\int_{\Sigma}\left(\rho_{k}-1\right)\left|\widetilde{u}_{k}\right|^{p_{k}-1} \widetilde{u}_{k} \varphi d X+\int_{\mathbb{R}^{2}}\left(\left|\widetilde{u}_{k}\right|^{p_{k}-1}-1\right) \widetilde{u}_{k} \varphi d X
\end{aligned}
$$

where the first integral goes to $\int_{\Sigma}(\nabla \widetilde{u} \nabla \varphi-\widetilde{u} \varphi) d X$ by the weak convergence of $\widetilde{u}_{k}$, the second one vanishes by the locally uniform convergence of $\rho$ and the boundedness of $\widetilde{u}_{k}$, and the third one vanishes too as it can be estimated by

$$
\begin{array}{r}
\left|\int_{\Sigma}\left(\left|\widetilde{u}_{k}\right|^{p_{k}-1}-1\right) \tilde{u}_{k} \varphi d X\right| \underset{\Sigma(2.4)}{=}\left(p_{k}-1\right) \int_{\Sigma} \int_{0}^{1}\left|\widetilde{u}_{k}\right|^{t\left(p_{k}-1\right)} d t\left|\widetilde{u}_{k}\right| \log \left|\widetilde{u}_{k}\right||\varphi| d X \\
\leq c\left(p_{k}-1\right) \int_{\Sigma}|\varphi| d X .
\end{array}
$$

If in addition $\Sigma$ can be taken such that $O \in \Sigma$, then $\widetilde{u}$ is nontrivial because clearly $\widetilde{u}(O)=1$. In that case the zero-set of $\widetilde{u}$ is made up by regular curves that may intersect only at some isolated points, see, for instance, [12]. Therefore

$$
\int_{\Sigma} p_{k} \rho_{k}\left|\widetilde{u}_{k}\right|^{p_{k}-1} \varphi d X \rightarrow \int_{\Sigma} \varphi d X
$$

for every function $\varphi \in C_{0}(\Sigma)$.

Case 1: $\bar{P} \in S_{n}$. In this case looking at (4.7)-(4.9) one sees that $\Sigma_{k}$ invades $\mathbb{R}^{2}$, so that $\widetilde{u}$ is a nontrivial solution of (4.12) with $\Sigma=\mathbb{R}^{2}$. On the other hand by Lemma 4.6, item (1) there exist at least $m+1$ linearly independent $n$-invariant functions $\phi_{j}$ with compact support such that $Q_{\mathbb{R}^{2}}^{0}\left(\phi_{j}\right)<0$. So (4.13) implies

$$
\int_{\Sigma_{k}}\left(\left|\nabla \phi_{j}\right|^{2}-p_{k} \rho_{k}\left|\widetilde{u}_{k}\right|^{p_{k}-1} \phi_{j}^{2}\right) d X<0
$$

Next we come back according to the change of variables (4.6) and define the functions $w_{j}\left(X^{\prime}\right)=\phi_{j}(X)$ as $X^{\prime} \in S_{n}$. They belong to the space $\mathcal{C}_{n}$ for large $k$ since their support is contained in a ball of radius $R / M_{k}$ centered at $P_{k}$ with $P_{k} \rightarrow \bar{P} \in S_{n}$. Moreover they are linearly independent and satisfy

$$
\begin{array}{r}
\int_{S_{n}}\left(\left|\nabla w_{j}\left(X^{\prime}\right)\right|^{2}-p_{k}\left|X^{\prime}\right|^{\alpha}\left|U_{p_{k}, n}\left(X^{\prime}\right)\right|^{p_{k}-1} w_{j}^{2}\left(X^{\prime}\right)\right) d X^{\prime}= \\
\int_{S_{n}}\left(\left|\nabla\left(\phi_{j}\left(M_{k}\left(X^{\prime}-P_{k}\right)\right)\right)\right|^{2}-p_{k} M_{k}^{2} \frac{\left|X^{\prime}\right|^{\alpha}}{|\bar{P}|^{\alpha}}\left|\widetilde{u}_{k}\left(M_{k}\left(X^{\prime}-P_{k}\right)\right)\right|^{p_{k}-1} \phi_{j}^{2}\left(M_{k}\left(X^{\prime}-P_{k}\right)\right)\right) d X^{\prime} \\
=\int_{\Sigma_{k}}\left(\left|\nabla \phi_{j}(X)\right|^{2}-p_{k} \rho_{k}(X)\left|\widetilde{u}_{k}(X)\right|^{p_{k}-1} \phi_{j}^{2}(X)\right) d X<0,
\end{array}
$$

which contradicts Lemma 4.5

Case 2: $\bar{P} \in \Gamma_{1}$. Different situations present depending if $\operatorname{dist}\left(P_{k}, \Gamma_{1}\right) M_{k} \rightarrow \infty$ or $\operatorname{dist}\left(P_{k}, \Gamma_{1}\right) M_{k} \rightarrow s>0$. Indeed $\operatorname{dist}\left(P_{k}, \Gamma_{1}\right) M_{k}$ cannot vanish because of the elliptic regularity up to the boundary, see [16, Case 2 in the proof of Theorem 1.1].

Case 2.a. If $\operatorname{dist}\left(P_{k}, \Gamma_{1}\right) M_{k} \rightarrow \infty$, looking at 4.7)-4.9) one sees that $\Sigma_{k}$ invades $\mathbb{R}^{2}$ and the conclusion follows as in Case 1 . 
Case 2.b. If $\operatorname{dist}\left(P_{k}, \Gamma_{1}\right) M_{k} \rightarrow s>0$, then for every $X \in \mathbb{R}^{2}$ we have

$$
M_{k}\left(\left|\frac{X}{M_{k}}+P_{k}\right|^{2}-1\right)=\frac{|X|^{2}}{M_{k}}+2 X \cdot P_{k}-\left(1+\left|P_{k}\right|\right) M_{k} \operatorname{dist}\left(P_{k}, \Gamma_{1}\right) \rightarrow 2(X-s \bar{P}) \cdot \bar{P} .
$$

So recalling (4.7) one sees that the curve $\Gamma_{1, k}$ goes to the straight line $\{X:(X-s \bar{P}) \cdot \bar{P}=0\}$, and $\Sigma_{k}$ invades the half-plane $\Sigma=\{X:(X-s \bar{P}) \cdot \bar{P}<0\}$. Because $s>0$, then $O \in \Sigma$ and $\widetilde{u}$ is a nontrivial solution to (4.12).

Now by Lemma 4.6. item (2) and Remark 4.7 there are $m+1$ linearly independent functions $\phi_{j}$ which are zero outside $B_{R} \cap\{X:(X-s \bar{P}) \cdot \bar{P} \leq-\delta\}$ such that $Q_{\Sigma}^{0}\left(\phi_{j}\right)<0$. In particular, for $k$ sufficiently large, their support is contained in $\Sigma_{k}$ and by the weak convergence of $p_{k} \rho_{k}\left|\widetilde{u}_{k}\right|^{p_{k}-1}$ we infer that

$$
\int_{\Sigma_{k}}\left(\left|\nabla \phi_{j}\right|^{2}-p_{k} \rho_{k}\left|\widetilde{u}_{k}\right|^{p_{k}-1} \phi_{j}^{2}\right) d X<0
$$

Eventually the functions $w_{j}\left(X^{\prime}\right)=\phi_{j}(X)$ as $X^{\prime} \in S_{n}$ belong to the space $\mathcal{C}_{n}$ for large $k$, because their supports do not touch the boundary of $S_{n}$. Moreover they are linearly independent and by the change of variables (4.6) one sees that

$$
\int_{S_{n}}\left(\left|\nabla w_{j}\right|^{2}-p_{k}\left|X^{\prime}\right|^{\alpha}\left|u_{k}\right|^{p_{k}-1} w_{j}^{2}\right) d X^{\prime}=\int_{\Sigma_{k}}\left(\left|\nabla \phi_{j}\right|^{2}-p_{k} \rho_{k}\left|\widetilde{u}_{k}\right|^{p_{k}-1} \phi_{j}^{2}\right) d X<0,
$$

which contradicts Lemma 4.5

Case 3: $\bar{P} \in \Gamma_{2} \cup \Gamma_{3}$. We only consider the case $\bar{P} \in \Gamma_{2}$, as the other one can be handled similarly. Now $\bar{P}=(\bar{x}, 0)$ for some $0<\bar{x}<1$ and $\operatorname{dist}\left(P_{k}, \Gamma_{2}\right)=y_{k}$ for large $k$. Again the limit set for $\Sigma_{k}$ changes according if either $y_{k} M_{k} \rightarrow \infty$ or to some $t \geq 0$.

Case 3.a. If $y_{k} M_{k} \rightarrow \infty, \Sigma_{k}$ invades $\mathbb{R}^{2}$ and the conclusion follows as in Case 1 .

Case 3.b. If $y_{k} M_{k} \rightarrow t \geq 0$, looking at (4.8) one sees that the segment $\Gamma_{2, k}$ goes to the straight line $y=-t$, and $\Sigma_{k}$ invades the half-plane $\{(x, y): y>-t\}$. So instead of $S_{n}$ we focus into a sector of amplitude $2 \pi / n$, namely

$$
S_{n}^{\sharp}=\left\{X=(x, y) \in \mathbb{R}^{2}:(x, y) \text { or }(x,-y) \in S_{n}\right\},
$$

and we slightly modify the scaling by taking

$$
\begin{array}{r}
X^{\prime}=\left(x^{\prime}, y^{\prime}\right) \quad \text { given by } x^{\prime}=x_{k}+\frac{x}{M_{k}}, y^{\prime}=\frac{y}{M_{k}}, \quad \text { as } X=(x, y) \\
\widetilde{u}_{k}(X)=\frac{u_{k}\left(X^{\prime}\right)}{\left\|u_{k}\right\|_{\infty}} \quad \text { for } X \in \Sigma_{k}^{\sharp}=\left\{X: X^{\prime} \in S_{n}^{\sharp}\right\}
\end{array}
$$

instead of (4.6). Minor changes to the previous arguments yield that $\Sigma_{k}^{\sharp}$ covers $\mathbb{R}^{2}$ and $\widetilde{u}_{k}$ converges in $C_{\text {loc }}\left(\mathbb{R}^{2}\right)$ to a solution of (4.12) in $\mathbb{R}^{2}$. The locally uniform convergence ensures that $\widetilde{u}(0, t)=1$ (because $\widetilde{u}_{k}\left(0, y_{k} M_{k}\right)=1$ with $\left.y_{k} M_{k} \rightarrow t\right)$, so that $\widetilde{u}$ is not identically zero and then also (4.13) holds true. Thank to this the same arguments used in Case 1 give that

$$
\int_{S_{n}^{\sharp}}\left(\left|\nabla w_{j}\right|^{2}-p_{k}\left|X^{\prime}\right|^{\alpha}\left|u_{k}\right|^{p_{k}-1} w_{j}^{2}\right) d X^{\prime}<0,
$$

where $w_{j}\left(X^{\prime}\right)=\phi_{j}(X)$, and $\phi_{j}$ are the functions produced in Lemma 4.6 item (1). In particular, due to the modified change of variables (4.14), both $u_{k}$ and $w_{j}$ are even w.r.t. the $y^{\prime}$ variable, therefore also

$$
\int_{S_{n}}\left(\left|\nabla w_{j}\right|^{2}-p_{k}\left|X^{\prime}\right|^{\alpha}\left|u_{k}\right|^{p_{k}-1} w_{j}^{2}\right) d X^{\prime}<0
$$

and $\partial_{y} w_{j}\left(x^{\prime}, 0\right)=0$, i.e. $\partial_{\nu} w_{j}=0$ on $\Gamma_{2}$. So the functions $w_{j}$, restricted to $S_{n}$, belong to $\mathcal{C}_{n}$ (because their support does not touch $\Gamma_{1}$ or $\Gamma_{3}$ if $k$ is large enough) and provide a contradiction with Lemma 4.5 
Case 4: $\bar{P}=A$ or $B$. We take $\bar{P}=B$, as the case $\bar{P}=A$ is similar. Now

$$
\operatorname{dist}\left(P_{k}, \partial S_{n}\right)=\min \left\{\operatorname{dist}\left(P_{k}, \partial \Gamma_{1}\right), \operatorname{dist}\left(P_{k}, \partial \Gamma_{2}\right)\right\} .
$$

If $\operatorname{dist}\left(P_{k}, \partial S_{n}\right) M_{k} \rightarrow \infty$ the conclusion follows as in Case 1. Otherwise we have to distinguish between different occurrencies:

Case 4.a: $\operatorname{dist}\left(P_{k}, \partial \Gamma_{1}\right) M_{k} \rightarrow s>0$ and $\operatorname{dist}\left(P_{k}, \partial \Gamma_{2}\right) M_{k} \rightarrow \infty$,

Case 4.b: $\operatorname{dist}\left(P_{k}, \partial \Gamma_{1}\right) M_{k} \rightarrow \infty$ and $\operatorname{dist}\left(P_{k}, \partial \Gamma_{2}\right) M_{k} \rightarrow t \geq 0$,

Case 4.c: $\operatorname{dist}\left(P_{k}, \partial \Gamma_{1}\right) M_{k} \rightarrow s>0$ and $\operatorname{dist}\left(P_{k}, \partial \Gamma_{2}\right) M_{k} \rightarrow t \geq 0$.

Indeed the occurrence $\operatorname{dist}\left(P_{k}, \partial \Gamma_{1}\right) M_{k} \rightarrow 0$ can not happen by the considerations in [16. Case 4.a and 4.b can be ruled out as we have done for Cases 2.b and 3.b, respectively.

As for Case 4.c, using the change of variables (4.14) the sets $\Sigma_{k}^{\sharp}$ cover the half-plane $\Sigma=\{(x, y): x<s\}$ and the functions $\widetilde{u}_{k}$ converge in $C_{\text {loc }}^{1}(\Sigma)$ to a solution of (4.12) on $\Sigma$, which is nontrivial since $u_{k}\left(0, y_{k} M_{k}\right)=1$ with $\left(0, y_{k} M_{k}\right) \rightarrow(0, t) \in \Sigma$ as $s>0$. Next the functions $\phi_{j}$ produced in Lemma 4.6, item (2) have support compactly contained in $\Sigma_{k}^{\sharp}$ and so

$$
\int_{\Sigma_{k}^{\sharp}}\left(\left|\nabla \phi_{j}\right|^{2}-p_{k} \rho_{k}\left|\widetilde{u}_{k}\right|^{p_{k}-1} \phi_{j}^{2}\right) d X<0
$$

by the weak convergence (4.13). Taking $w_{j}\left(X^{\prime}\right)=\phi_{j}(X)$, one can easily see that

$$
\int_{S_{n}^{\sharp}}\left(\left|\nabla w_{j}\right|^{2}-p_{k}\left|X^{\prime}\right|^{\alpha}\left|u_{k}\right|^{p_{k}-1} w_{j}^{2}\right) d X^{\prime}<0 .
$$

But, due to the modified change of variables (4.14), $w_{j}$ are even w.r.t. the $y^{\prime}$ variable, and certainly the same holds for $u_{k}$. Therefore also

$$
\int_{S_{n}}\left(\left|\nabla w_{j}\right|^{2}-p_{k}\left|X^{\prime}\right|^{\alpha}\left|u_{k}\right|^{p_{k}-1} w_{j}^{2}\right) d X^{\prime}<0
$$

and by symmetry on $\Gamma_{2} \subset\left\{\left(x^{\prime}, y^{\prime}\right): y^{\prime}=0\right\}$ we have $\partial_{\nu} w_{j}=\partial_{y} w_{j}\left(y^{\prime}, 0\right)=0$. Eventually the restriction of $w_{j}$ to $\left\{\left(x^{\prime}, y^{\prime}\right): y^{\prime}>0\right\}$ belongs to $\mathcal{C}_{n}$, because its support does not touch either $\Gamma_{1}$ or $\Gamma_{3}$ if $k$ is taken sufficiently large, and this concludes this part of the proof.

Case 5: $\bar{P}=O$. Here we need a different scaling parameter because $M_{k}$ given in 4.5] is constantly 0 .

Case 5.1: $\bar{P}=O$ and $\left|P_{k}\right|^{2+\alpha}\left\|u_{k}\right\|_{\infty}^{p_{k}-1} \rightarrow \infty$. We take

$$
M_{k}=\left|P_{k}\right|^{\frac{\alpha}{2}}\left\|u_{k}\right\|_{\infty}^{\frac{p_{k}-1}{\infty^{2}}}
$$

so that $M_{k}=\frac{\left|P_{k}\right|^{\frac{2+\alpha}{2}}\left\|u_{k}\right\|_{\infty}^{\frac{p_{k}-1}{2}}}{\left|P_{k}\right|} \rightarrow \infty$. Next we use the same change of variables (4.6), but with a different value for $M_{k}$. In that way $\widetilde{u}_{k}$ solves a problem of type (4.10) for

$$
\rho_{k}(X)=\left(\frac{\left|X^{\prime}\right|}{M_{k}\left|P_{k}\right|}\right)^{\alpha}=\left(\frac{\left|M_{k} P_{k}+X\right|}{M_{k}\left|P_{k}\right|}\right)^{\alpha},
$$

which converges to 1 locally uniformly because also $M_{k}\left|P_{k}\right| \rightarrow \infty$.

Concerning the limit set for $\Sigma_{k}$, it changes depending if $\operatorname{dist}\left(P_{k}, \partial \Sigma_{k}\right) M_{k}$ is bounded or not. If $\operatorname{dist}\left(P_{k}, \partial \Sigma_{k}\right) M_{k} \rightarrow \infty$, then $\Sigma_{k}$ invades $\mathbb{R}^{2}$ and we can conclude as in Case 1.

Otherwise if $\operatorname{dist}\left(P_{k}, \partial \Sigma_{k}\right) M_{k} \rightarrow t \geq 0$, we remark that

$$
\operatorname{dist}\left(P_{k}, \partial \Sigma_{k}\right)=\min \left\{\operatorname{dist}\left(P_{k}, \Gamma_{2, k}\right), \operatorname{dist}\left(P_{k}, \partial \Gamma_{3, k}\right)\right\} .
$$

To fix ideas we take that $\operatorname{dist}\left(P_{k}, \partial \Sigma_{k}\right)=\operatorname{dist}\left(P_{k}, \Gamma_{2, k}\right)=y_{k}$ along a subsequence (the opposite case can be dealt in similarly). In the present situation $y_{k} M_{k}$ is bounded and $\left|P_{k}\right| M_{k} \rightarrow \infty$, therefore $x_{k} M_{k} \rightarrow \infty$. Consequently $\Sigma_{k}$ goes to the half-space $\{y>-t\}$, and one can reason as in Case 4.b. 
Case 5.2: $\bar{P}=O$ and $\left|P_{k}\right|^{2+\alpha}\left\|u_{k}\right\|_{\infty}^{p_{k}-1}$ is bounded.

We chose as a scaling parameter

$$
\mu_{k}=\left\|u_{k}\right\|_{\infty}^{\frac{p_{k}-1}{2+\alpha}}
$$

and define

$$
\widehat{u}_{k}(X)=\frac{1}{\left\|u_{k}\right\|_{\infty}} u_{k}\left(X / \mu_{k}\right) \quad \text { in } B_{k}=\left\{X:|X|<\mu_{k}\right\}
$$

which solves

$$
\begin{cases}-\Delta \widehat{u}_{k}=|X|^{\alpha}\left|\widehat{u}_{k}\right|^{p_{k}-1} \widehat{u}_{k} & \text { in } B_{k}, \\ \widehat{u}_{k}=0 & \text { on } \partial B_{k} .\end{cases}
$$

Now $\widehat{u}_{k}\left(\mu_{k} P_{k}\right)=1=\left\|\widehat{u}_{k}\right\|_{\infty}$ and $B_{k}$ invades $\mathbb{R}^{2}$, therefore one can see that also in this case $\widehat{u}_{k}$ converges locally uniformly to a solution of

$$
-\Delta \widehat{u}=|x|^{\alpha} \widehat{u} \quad x \in \mathbb{R}^{2} .
$$

Let us check that the function $\widehat{u}$ is nontrivial. Since $\mu_{k} P_{k}$ is bounded we can assume that it converges to some point $Q_{0}$, and by the locally uniform convergence $\widehat{u}\left(Q_{0}\right)=1$.

Eventually, take the $m+1$ linearly independent functions $\phi_{j}$ produced in Lemma 4.6 step (1) with compact support such that $Q_{\mathbb{R}^{2}}^{\alpha}\left(\phi_{j}\right)<0$. The convergence of $\widehat{u}_{k}$ yields that also

$$
\int_{B_{k}}\left(\left|\nabla \phi_{j}\right|^{2}-p_{k}|X|^{\alpha}\left|w_{k}\right|^{p_{k}-1} \phi_{j}^{2}\right) d X<0 .
$$

Eventually defining $\psi_{j}(X)=\phi_{j}\left(\mu_{k} X\right)$ for $X \in B$, we see that $\psi_{j} \in H_{0, n}^{1}$ (because the change of variable (4.16) does not break the symmetries) and

$$
\int_{B}\left(\left|\nabla \psi_{j}\right|^{2}-p_{k}|X|^{\alpha}\left|u_{k}\right|^{p_{k}-1} \psi_{j}^{2}\right) d X=\int_{B_{k}}\left(\left|\nabla \phi_{j}\right|^{2}-p_{k}|X|^{\alpha}\left|w_{k}\right|^{p_{k}-1} \phi_{j}^{2}\right) d X<0,
$$

which contradicts the fact that the $n$-Morse index of $u_{k}$ is at most $m$.

Thank to Lemma 4.2 and Proposition 4.8, we can apply Lemma 2.1 to any sequence $U_{p_{k}, n}$ of least energy $n$-invariant nodal solutions with $p_{k} \rightarrow 1$ and deduce that (2.1) and (2.6) hold for some eigenvalue $\mu_{j, i}$ of (1.3) and related eigenfunction $\omega_{j, i}$ (normalized so that $\left.\left\|\omega_{j, i}\right\|_{\infty}=1\right)$. Of course the index $(j, i)$ has to be selected in such a way that $\omega_{j, i} \in H_{0, n}^{1}$. So from the computation preformed in Lemma 2.3 we deduce that

$$
\begin{aligned}
\mu_{j, i} & =\left(\frac{2+\alpha}{2} z_{i}\left(\frac{2 j}{2+\alpha}\right)\right)^{2}, \\
\omega_{j, i}(r, \theta) & = \pm \frac{1}{\left\|\mathcal{J}_{\frac{2 j}{2+\alpha}}\right\|_{\infty}} \mathcal{J}_{\frac{2 j}{2+\alpha}}\left(z_{i}\left(\frac{2 j}{2+\alpha}\right) r^{\frac{2+\alpha}{2}}\right) \cos (j \theta),
\end{aligned}
$$

where the index $j$ can be 0 or a multiple of $n$. Here we have also used that each Bessel function attains is global extremum in its first nodal interval and that in dimension $N=2$ the eigenfunctions of the Laplace-Beltrami operator are of type $A \cos (j \theta)+B \sin (j \theta)$, so that they belong to $H_{0, n}^{1}$ only when $j=0$ or $j$ is a multiple of $n$ and $B$ is zero.

Next we see that the minimality of $U_{p, n}$ implies that $\mu_{j, i}$ must be the second eigenvalue of (1.3) in the space $H_{0, n}^{1}$, and so we single out the exact value of $j$ and $i$. This gives Theorem 1.3

Proof of Theorem 1.3. With a little abuse of notation, we write $p \rightarrow 1$ meaning any sequence $p_{k} \rightarrow 1$. We have already pointed out that $\left\|U_{p, n}\right\|_{\infty}^{p-1}$ and $\bar{U}_{p}:=U_{p, n} /\left\|U_{p, n}\right\|_{\infty}$ converge respectively to an eigenvalue $\mu_{j, i}$ and an eigenfuntion $\omega_{j, i}$ of (1.3) described by (4.17) and (4.18). It remains to check that the values of $j$ and $i$ in (2.1) are $n$ and 1 (or 0 and 2) if $n<\frac{2+\alpha}{2} \beta$ (or else $n>\frac{2+\alpha}{2} \beta$ ). We divide the proof of this fact in several steps. 
Step 1: $\mu_{j, i}>\mu_{0,1}$.

First we observe that $\bar{u}_{p}$ cannot go to the first eigenfunction $\omega_{0,1}$. Otherwise (2.1) assures that $p\left\|U_{p, n}\right\|_{\infty}^{p-1} \rightarrow \mu_{0,1}<\mu_{h, \ell}$ for every $(h, \ell) \neq(0,1)$, since the first eigenvalue is simple. Letting $\mu$ be the second eigenvalue of (1.3) in $H_{0, n}^{1}$, for every $w \in H_{0, n}^{1}, w \perp \omega_{0,1}$ we have

$$
\begin{aligned}
\mathcal{Q}_{U_{p, n}}(w) & =\int_{B}|\nabla w|^{2} d x-\int_{B} p|x|^{\alpha}\left|U_{p, n}\right|^{p-1} w^{2} d x \geq \mu \int_{B}|x|^{\alpha} w^{2} d x-\int_{B} p|x|^{\alpha}\left|U_{p, n}\right|^{p-1} w^{2} d x \\
& \geq\left(\mu-p\left\|U_{p, n}\right\|_{\infty}^{p-1}\right) \int_{B}|x|^{\alpha}|w|^{2} d x \geq \varepsilon \int_{B}|x|^{\alpha}|w|^{2} d x
\end{aligned}
$$

for some $\varepsilon>0$ when $p$ is close to 1 . Hence in this case the $n$-Morse index of $U_{p, n}$ would be at most 1 , contradicting Lemma 4.2 .

Step 2: $\mu_{j, i} \leq \mu_{n, 1}$.

By the minimality of $U_{p, n}$, and observing that $\mathcal{E}_{p}(v)=\frac{p-1}{2(p+1)} \int_{B}|\nabla v|^{2} d x$ for any function $v \in \mathcal{N}_{p, n}^{\text {nod }}$, we have

$$
\int_{B}\left|\nabla \bar{U}_{p, n}\right|^{2} d x=\frac{1}{\left\|U_{p, n}\right\|_{\infty}^{2}} \int_{B}\left|\nabla U_{p, n}\right|^{2} d x \leq \frac{1}{\left\|U_{p, n}\right\|_{\infty}^{2}} \int_{B}|\nabla v|^{2} d x
$$

for every $v \in \mathcal{N}_{p, n}^{\text {nod }}$. So (2.1) implies that

$$
0<\int_{B}\left|\nabla \omega_{j, i}\right|^{2} d x \leq \liminf _{p \rightarrow 1} \frac{1}{\left\|U_{p, n}\right\|_{\infty}^{2}} \int_{B}\left|\nabla v_{p}\right|^{2} d x
$$

for every sequence $v_{p}$ in $\mathcal{N}_{p, n}^{\text {nod }}$.

Next we define

$$
v_{p}:=A_{p} \omega_{n, 1}
$$

where $\omega_{n, 1}$ is defined according to (4.18), and check that we can chose the constant $A_{p}>0$ in such a way that $v_{p} \in \mathcal{N}_{p, n}^{\text {nod }}$ for every $p$.

The support of the positive/negative parts of $v_{p}$ are $B^{ \pm}=\bigcup_{i=0}^{n-1} \Sigma_{i}^{ \pm}$for $\Sigma_{i}^{+}=\{(r, \theta): 0 \leq$ $\left.r \leq 1, \frac{2 i-1}{2 n} \pi \leq \theta \leq \frac{2 i+1}{2 n} \pi\right\}$ and $\Sigma_{i}^{-}=\left\{(r, \theta): 0 \leq r \leq 1, \frac{2 i+1}{2 n} \pi \leq \theta \leq \frac{2 i+3}{2} \pi\right\}$, so that by periodicity

$\int_{B}|x|^{\alpha}\left|v_{p}^{+}\right|^{p+1} d x=A_{p}^{p+1} \int_{B}|x|^{\alpha}\left|\omega_{n, 1}^{+}\right|^{p+1} d x=n A_{p}^{p+1} \int_{\Sigma_{0}^{+}}|x|^{\alpha}\left|\omega_{n, 1}\right|^{p+1} d x$

and by simmetry

$$
=n A_{p}^{p+1} \int_{\Sigma_{0}^{-}}|x|^{\alpha}\left|\omega_{n, 1}\right|^{p+1} d x=A_{p}^{p+1} \int_{B}|x|^{\alpha}\left|\omega_{n, 1}^{-}\right|^{p+1} d x=\int_{B}|x|^{\alpha}\left|v_{p}^{-}\right|^{p+1} d x .
$$

On the other hand $v_{p}$ is an eigenfunction for (1.3) related to $\mu_{n, 1}$, hence

$$
\int_{B}\left|\nabla v_{p}^{ \pm}\right|^{2} d x=\int_{B} \nabla v_{p} \nabla v^{ \pm} d x=\mu_{n, 1} \int_{B}|x|^{\alpha} v_{p} v_{p}^{ \pm} d x=\mu_{n, 1} \int_{B}|x|^{\alpha}\left|v_{p}^{ \pm}\right|^{2} d x
$$

and by virtue of the periodicity and simmetry of $\cos (n \theta)$ we get

Summing up, $v_{p} \in \mathcal{N}_{n, \operatorname{nod}}$ provided that

$$
\begin{aligned}
\int_{B}\left|\nabla v_{p}^{+}\right|^{2} d x & =A_{p}^{2} \mu_{n, 1} \int_{B}|x|^{\alpha}\left|\omega_{n, 1}^{+}\right|^{2} d x=\frac{1}{2} A_{p}^{2} \mu_{n, 1} \int_{B}|x|^{\alpha}\left|\omega_{n, 1}\right|^{2} d x \\
& =A_{p}^{2} \mu_{n, 1} \int_{B}|x|^{\alpha}\left|\omega_{n, 1}^{-}\right|^{2} d x=\int_{B}\left|\nabla v_{p}^{-}\right|^{2} d x
\end{aligned}
$$

$$
A_{p}=\left(\mu_{n, 1} \frac{\int_{B}|x|^{\alpha}\left|\omega_{n, 1}^{ \pm}\right|^{2} d x}{\int_{B}|x|^{\alpha}\left|\omega_{n, 1}^{ \pm}\right|^{p+1} d x}\right)^{\frac{1}{p-1}}=\left(\mu_{n, 1} \frac{\int_{B}|x|^{\alpha}\left|\omega_{n, 1}\right|^{2} d x}{\int_{B}|x|^{\alpha}\left|\omega_{n, 1}\right|^{p+1} d x}\right)^{\frac{1}{p-1}}
$$


Inserting $v_{p}$ into (4.19) gives

$$
0<\liminf _{p \rightarrow 1} \frac{1}{\left\|U_{p, n}\right\|_{\infty}^{2}} \int_{B}\left|\nabla v_{p}\right|^{2} d x=\liminf _{p \rightarrow 1}\left(\frac{A_{p}}{\left\|U_{p, n}\right\|_{\infty}}\right)^{2} \int_{B}\left|\nabla \omega_{n, 1}\right|^{2} d x
$$

and because $\omega_{n, 1}$ is an eigenfunction for (1.3) related to $\mu_{n, 1}$

$$
=\liminf _{p \rightarrow 1}\left(\frac{A_{p}}{\left\|U_{p, n}\right\|_{\infty}}\right)^{2} \mu_{n, 1} \int_{B}|x|^{\alpha}\left|\omega_{n, 1}\right|^{2} d x
$$

and (4.20) gives

$$
=\liminf _{p \rightarrow 1}\left(\frac{\mu_{n, 1}^{\frac{p+1}{2}}}{\left\|U_{p, n}\right\|_{\infty}^{p-1}} \frac{\left(\int_{B}|x|^{\alpha}\left|\omega_{n, 1}\right|^{2} d x\right)^{\frac{p+1}{2}}}{\int_{B}|x|^{\alpha}\left|\omega_{n, 1}\right|^{p+1} d x}\right)^{\frac{2}{p-1}} .
$$

Since $1 /(p-1) \rightarrow \infty$, a necessary condition is

$$
1 \leq \liminf _{p \rightarrow 1} \frac{\mu_{n, 1}^{\frac{p+1}{2}}}{\left\|U_{p, n}\right\|_{\infty}^{p-1}} \frac{\left(\int_{B}|x|^{\alpha}\left|\omega_{n, 1}\right|^{2} d x\right)^{\frac{p+1}{2}}}{\int_{B}|x|^{\alpha}\left|\omega_{n, 1}\right|^{p+1} d x}=\frac{\mu_{n, 1}}{\mu_{j, i}}
$$

by (2.1), which implies $\mu_{j, i} \leq \mu_{n, 1}$.

Step 3: $\mu_{j, i} \leq \mu_{0,2}$.

We follow the same line of Step 2 and define

$$
v_{p}(x):= \begin{cases}A_{p}^{+} \omega_{0,2}(x)=A_{p}^{+} \mathcal{J}_{0}\left(z_{2}(0)|x|^{\frac{2+\alpha}{2}}\right) & \text { if }|x| \leq R:=\left(\frac{z_{1}(0)}{z_{2}(0)}\right)^{\frac{2}{2+\alpha}} \\ A_{p}^{-} \omega_{0,2}(x)=A_{p}^{-} \mathcal{J}_{0}\left(z_{2}(0)|x|^{\frac{2+\alpha}{2}}\right) & \text { if } R<|x| \leq 1,\end{cases}
$$

and choose the constants $A_{p}^{ \pm}>0$ in such a way that $v_{p} \in \mathcal{N}_{p, n}^{\text {nod }}$ for every $p$. First notice that since $v_{p}(x)=0$ if and only if $|x|=R$, then $v_{p} \in H_{0}^{1}(B)$ and the support of its positive/negative parts are respectively $\Omega^{+}=B_{R}$ and $\Omega^{-}=B \backslash B_{R}$. Next

$$
\begin{gathered}
\int_{B}|x|^{\alpha}\left|v_{p}^{ \pm}\right|^{p+1} d x=\left(A_{p}^{ \pm}\right)^{p+1} \int_{\Omega^{ \pm}}|x|^{\alpha}\left|\omega_{0,2}\right|^{p+1} d x, \\
\int_{B}\left|\nabla v_{p}^{ \pm}\right|^{2} d x=\left(A_{p}^{ \pm}\right)^{2} \int_{B}\left|\nabla \omega_{0,2}^{ \pm}\right|^{2} d x=\left(A_{p}^{ \pm}\right)^{2} \int_{B} \nabla \omega_{0,2} \nabla \omega_{0,2}^{ \pm} d x
\end{gathered}
$$

and since $\omega_{0,2}$ is an eigenfunction for (1.3) related to $\mu_{0,2}$, using $\left(A_{p}^{ \pm}\right)^{2} \omega_{0,2}^{ \pm} \in H_{0}^{1}(B)$ as a test function gives

$$
=\mu_{0,2}\left(A_{p}^{ \pm}\right)^{2} \int_{B}|x|^{\alpha} \omega_{0,2} \omega_{0,2}^{ \pm} d x=\mu_{0,2}\left(A_{p}^{ \pm}\right)^{2} \int_{B}|x|^{\alpha}\left|\omega_{0,2}^{ \pm}\right|^{2} d x
$$

Summing up, $v_{p} \in \mathcal{N}_{n, \text { nod }}$ provided that

$$
A_{p}^{ \pm}=\left(\mu_{0,2} \frac{\int_{B}|x|^{\alpha}\left|\omega_{0,2}^{ \pm}\right|^{2} d x}{\int_{B}|x|^{\alpha}\left|\omega_{0,2}^{ \pm}\right|^{p+1} d x}\right)^{\frac{1}{p-1}}
$$

Inserting $v_{p}$ into (4.19) gives

$0<\liminf _{p \rightarrow 1} \frac{1}{\left\|U_{p, n}\right\|_{\infty}^{2}} \int_{B}\left|\nabla v_{p}\right|^{2} d x=\liminf _{p \rightarrow 1} \frac{1}{\left\|U_{p, n}\right\|_{\infty}^{2}}\left[\left(A_{p}^{+}\right)^{2} \int_{B}\left|\nabla \omega_{n, 1}^{+}\right|^{2} d x+\left(A_{p}^{-}\right)^{2} \int_{B}\left|\nabla \omega_{n, 1}^{-}\right|^{2} d x\right]$ 
and by the previous observations

$$
=\liminf _{p \rightarrow 1} \frac{\mu_{0,2}}{\left\|U_{p, n}\right\|_{\infty}^{2}}\left[\left(A_{p}^{+}\right)^{2} \int_{B}|x|^{\alpha}\left|\omega_{n, 1}^{+}\right|^{2} d x+\left(A_{p}^{-}\right)^{2} \int_{B}|x|^{\alpha}\left|\omega_{n, 1}^{-}\right|^{2} d x\right]
$$

next using 4.21) gives

$$
=\liminf _{p \rightarrow 1}\left(\frac{\mu_{0,2}^{\frac{p+1}{2}}}{\left\|U_{p, n}\right\|_{\infty}^{p-1}}\right)^{\frac{2}{p-1}}\left[\left(\frac{\left(\int_{B}|x|^{\alpha}\left|\omega_{2,0}^{+}\right|^{2} d x\right)^{\frac{p+1}{2}}}{\int_{B}|x|^{\alpha}\left|\omega_{2,0}^{+}\right|^{p+1} d x}\right)^{\frac{2}{p-1}}+\left(\frac{\left(\int_{B}|x|^{\alpha}\left|\omega_{2,0}^{-}\right|^{2} d x\right)^{\frac{p+1}{2}}}{\int_{B}|x|^{\alpha}\left|\omega_{2,0}^{-}\right|^{p+1} d x}\right)^{\frac{2}{p-1}}\right]
$$

Since $1 /(p-1) \rightarrow \infty$, a necessary condition is that or

$$
1 \leq \liminf _{p \rightarrow 1} \frac{\mu_{0,2}^{\frac{p+1}{2}}}{\left\|U_{p, n}\right\|_{\infty}^{p-1}} \frac{\left(\int_{B}|x|^{\alpha}\left|\omega_{0,2}^{ \pm}\right|^{2} d x\right)^{\frac{p+1}{2}}}{\int_{B}|x|^{\alpha}\left|\omega_{0,2}^{ \pm}\right|^{p+1} d x}=\frac{\mu_{0,2}}{\mu_{j, i}}
$$

so that also in this case $\mu_{j, i} \leq \mu_{0,2}$.

Step 4: The second eigenvalue of (1.3) in $H_{0, n}^{1}$ is simple whenever $n \neq \frac{2+\alpha}{2} \beta$, and precisely it is given by $\mu_{n, 1}$ or $\mu_{0,2}$ depending if $n \lessgtr \frac{2+\alpha}{2} \beta$.

Thanks to (4.17) it is equivalent to see that the following inequalities hold among the zeros of different Bessel functions:

$$
\begin{gathered}
z_{1}(0)<z_{1}\left(\frac{2 n}{2+\alpha}\right)<z_{1}\left(\frac{2 h n}{2+\alpha}\right) \quad \text { as } h \geq 2, \\
z_{2}(0) \gtrless z_{1}\left(\frac{2 n}{2+\alpha}\right) \quad \text { if } n \lessgtr \frac{2+\alpha}{2} \beta .
\end{gathered}
$$

They both are consequences of the fact that the map $\beta \mapsto z_{1}(\beta)$ is increasing. The first one is trivial, while the second one holds true because by definition of $\beta$ we have $z_{2}(0)=$ $z_{1}(\beta) \gtrless z_{1}\left(\frac{2 n}{2+\alpha}\right)$ according if $\beta \gtrless \frac{2 n}{2+\alpha}$.

Step 5: conclusion.

By Steps 1-3 we know that $\mu_{0,1}<\mu_{j, i} \leq \min \left\{\mu_{n, 1}, \mu_{0,2}\right\}$. Next Step 4 guarantees that there are not eigenvalues in the range $\left(\mu_{0,1}, \min \left\{\mu_{n, 1}, \mu_{0,2}\right\}\right)$, therefore $\mu_{j, i}=\min \left\{\mu_{n, 1}, \mu_{0,2}\right\}=$ $\mu_{n, 1}$ if $n<\frac{2+\alpha}{2} \beta$, or $\mu_{0,2}$ otherwise. So, remembering that the second eigenvalue is simple unless $n=\frac{2+\alpha}{2} \beta$, we have proved that (2.1) holds for $(j, i)=(n, 1)$ if $n<\frac{2+\alpha}{2} \beta$, or else for $(j, i)=(0,2)$ when $n>\frac{2+\alpha}{2} \beta$.

Remark 4.9. In the particular case $n=\frac{2+\alpha}{2} \beta$, then $z_{1}\left(\frac{2 n}{2+\alpha}\right)=z_{1}(\beta)=z_{2}(0)$ by definition of $\beta$. Hence $\mu_{n, 1}=\mu_{0,2}$ has multiplicity two as the second eigenvalue of (1.3) in $H_{0, n}^{1}$, having both a radial and a nonradial eigenfunction. (1.10) and (1.12) are equivalent and hold true, but we are not able to deduce the asymptotic behaviour of $U_{p, n}$.

4.2. Proof of the multiplicity result. From the asymptotic profile in Theorem 1.3, it is not hard to see that for $p$ close to 1 the least energy nodal $n$-invariant solutions $U_{p, n}$ are nonradial and different one from another for $n=1, \ldots\left\lceil\frac{2+\alpha}{2} \beta-1\right\rceil$, radial otherwise.

First we conclude the proof of Theorem 1.4

Proof of Theorem 1.4. We have seen in Corollary 4.4 that there exists $\bar{p}=\bar{p}(\alpha)$ such that $U_{p, n}$ is nonradial for $1<p<\bar{p}$, as $n=1, \ldots\left\lceil\frac{2+\alpha}{2} \beta-1\right\rceil$. It remains to check that $U_{p, n} \neq$ $U_{p, k}$ if $n \neq k$ for every $p$ in a right neighborhood of 1 , possibly smaller than $(1, \bar{p})$. It follows by Theorem 1.3 which states that they converge to different eigenfunctions of (1.3). 
After we show that the other least energy $n$-invariant solutions, i.e. $U_{p, n}$ for $n>\frac{2+\alpha}{2} \beta$, are radial for $p$ close to 1 , by adapting to the Hénon equation the arguments in [19, Proposition $10.5]$

Proposition 4.10. Let $n>\frac{2+\alpha}{2} \beta$, then there exists $\bar{p}>1$ such that for every $p \in(1, \bar{p})$ $U_{p, n}$ is radial and coincides with $u_{p}^{*}$.

Proof. In this case we know by Theorems 1.1 and 1.3 that both $\left\|u_{p}^{*}\right\|_{\infty}^{p-1}$ and $\left\|U_{p, n}\right\|_{\infty}^{p-1}$ converge to $\mu_{0,2}=\left(\frac{2+\alpha}{2} z_{2}(0)\right)^{2}$. We assume that for a given sequence $p_{k} \rightarrow 1 U_{p_{k}, n} \neq u_{p_{k}}^{*}$ and deduce a contradiction. To this aim we define

$$
w_{k}=\frac{U_{p_{k}, n}-u_{p_{k}}^{*}}{\left\|U_{p_{k}, n}-u_{p_{k}}^{*}\right\|_{\infty}} .
$$

The assumption $U_{p_{k}, n} \neq u_{p_{k}}^{*}$ implies that there is a sequence $P_{k} \in B$ where $w_{k}\left(P_{k}\right)= \pm 1$, and w.l.o.g. we can take $w_{k}\left(P_{k}\right)=1$ and $P_{k} \rightarrow \bar{P} \in \bar{B}$. Furthermore $w_{k}$ solves a linear Dirichlet problem

$$
\begin{cases}-\Delta w_{k}=|x|^{\alpha} p_{k} \mu_{0,2} c_{k} w_{k} & \text { in } B \\ w_{k}=0 & \text { on } \partial B\end{cases}
$$

where $c_{k}$ is given by the Mean Value Theorem

$$
c_{k}(x)=\frac{1}{\mu_{0,2}} \int_{0}^{1}\left|t U_{p_{k}, n}(x)+(1-t) u_{p_{k}}^{*}(x)\right|^{p_{k}-1} d t .
$$

Clearly $\left|c_{k}(x)\right| \leq C\left(\left\|U_{p_{k}, n}\right\|_{\infty}^{p_{k}-1}+\left\|u_{p_{k}}^{*}\right\|_{\infty}^{p_{k}-1}\right)$ is bounded, let us check that $c_{k}(x) \rightarrow 1$ almost everywhere. Indeed the asymptotic expansion in (2.7) for both $u_{p}^{*}$ and $U_{p, n}$ gives that

$$
h(x, t):=t \mu_{0,2}^{-\frac{1}{p_{k}-1}} U_{p_{k}, n}+(1-t) \mu_{0,2}^{-\frac{1}{p_{k}-1}} u_{p_{k}}^{*} \rightarrow e^{c} \omega_{0,2}
$$

uniformly for $(x, t) \in \bar{B} \times[0,1]$, where $c$ is the constant defined in (2.5). So

$$
c_{k}(x)=\int_{0}^{1}|h(x, t)|^{p_{k}-1} d t \rightarrow 1
$$

uniformly on any closed subsect of $B$ which does not contain the zero set of $\omega_{0,2}$, i.e. the circle of radius $z_{1}(0) / z_{2}(0)$. Therefore $w_{k}$ converges (weakly and then, by elliptic estimates, in $C(\bar{B})$ ) to a solution $w$ of (1.3) related to $\mu_{0,2}$. Such limit function is nontrivial since by the uniform convergence $w(\bar{P})=1=\|w\|_{\infty}$, hence Lemma 2.3 yields $w(x)=\omega_{0,2}(x)=$ $\mathcal{J}_{0}\left(z_{2}(0)|x|\right)$.

On the other multiplying the equation in (4.22) by $\omega_{0,2}$ and integrating by parts gives

$$
p_{k} \mu_{0,2} \int_{B}|x|^{\alpha} c_{k} w_{k} \omega_{0,2} d x=\int_{B} \nabla w_{k} \nabla \omega_{0,2} d x=\mu_{0,2} \int_{B}|x|^{\alpha} w_{k} \omega_{0,2} d x .
$$

So

$$
\left(p_{k}-1\right) \int_{B}|x|^{\alpha} c_{k} w_{k} \omega_{0,2} d x=\int_{B}|x|^{\alpha}\left(1-c_{k}\right) w_{k} \omega_{0,2} d x .
$$

But using the definition of $c_{k}$ and the elementary equality (2.4) one sees that

$$
c_{k}-1=\left(p_{k}-1\right) \int_{0}^{1} \log |h(t, x)| \int_{0}^{1}|h(t, x)|^{s\left(p_{k}-1\right)} d s d t
$$

which inserted into 4.25 gives

$$
\int_{B}|x|^{\alpha} c_{k} w_{k} \omega_{0,2} d x=-\int_{B}|x|^{\alpha} w_{k} \omega_{0,2} \int_{0}^{1} \log |h(t, x)| \int_{0}^{1}|h(t, x)|^{s\left(p_{k}-1\right)} d s d t d x
$$


and passing to the limit (4.23), 4.24) imply

$$
\int_{B}|x|^{\alpha} \omega_{0,2}^{2} d x=-\int_{B}|x|^{\alpha} \omega_{0,2}^{2}\left(c+\log \left|\omega_{0,2}\right|\right) d x=0
$$

by the definition of the constant $c$ given in (2.5). But of course $\omega_{0,2}$ is nontrivial, and so we have reached the desired contradiction.

In 7 it has been proved that for large values of $p$ the functions $U_{p, n}$ are nonradial for $n<\frac{2+\alpha}{2} \kappa$, where $\kappa \approx 5.1869$ is a fixed number related to the computation of the Morse index when $p \rightarrow \infty$. Therefore in the range $\left[\frac{2+\alpha}{2} \beta+1\right] \leq n \leq\left\lceil\frac{2+\alpha}{2} \kappa-1\right\rceil$ there is a breaking of symmetry, in the sense that the curve $p \mapsto U_{p, n}$ coincides with the curve of radial solution on an interval $\left(1, \bar{p}_{n}\right)$, and then bifurcates giving rise to a global branch of nonradial solutions. Asides from $n$-invariant solutions, the issue of nonradial bifurcation from the curves $p \mapsto u_{p}$ of radial solutions (even with a larger number of nodal zones, and in higher dimension) and the separation of the various branches deserves a further study, which can be carried on starting from the computation of the Morse index of radial solutions at the ends of the existence range performed here and in [6], 7]. It will be the object of a forthcoming paper [2].

\section{REFERENCES}

[1] A. Aftalion, F. Pacella, Qualitative properties of nodal solutions of semilinear elliptic equations in radially symmetric domains Comptes Rendus Mathematique, 339/5 (2004), 339-344. DOI: 10.1016/j.crma.2004.07.004

[2] A.L. Amadori, Nonradial sign-changing solutions for the Hénon equation by bifurcation, in preparation

[3] A.L. Amadori, F. Gladiali, Bifurcation and symmetry breaking for the Hénon equation, Advances in Differential Equations 19 (2014) n.7-8, 755-782, http://projecteuclid.org/euclid.ade/1399395725

[4] A.L. Amadori, F. Gladiali, On a singular eigenvalue problem and its applications in computing the Morse index of solutions to semilinear PDE's (2018) arXiv:

[5] A.L. Amadori, F. Gladiali, On a singular eigenvalue problem and its applications in computing the Morse index of solutions to semilinear PDE's, Part II (2019) arXiv:

[6] A.L. Amadori, F. Gladiali, Asymptotic profile and Morse index of nodal radial solutions to the Hénon problem, (2018) arXiv:1810.11046

[7] A.L. Amadori, F. Gladiali, The Hénon problem with large exponent in the disc, (2019) arXiv:1904.05907

[8] T. Bartsch, T. Weth, A note on additional properties of sign changing solutions to superlinear elliptic equations, Topological Methods in Nonlinear Analysis 22 (2003), 1-14.

[9] T. Bartsch, T. Weth, M. Willem, Partial symmetry of least energy nodal solutions to some variational problems, J. Anal. Math. 96 (2005), 1-18.

[10] T. Bartsch, M. Willem, Infinitely many radial solutions of a semilinear elliptic problem on $\mathbb{R}^{N}$ Archive for Rational Mechanics and Analysis, 124/3 (1993), 261-276. DOI: 10.1007/BF00953069

[11] Bonheure, D., Bouchez, V., Grumiau, C., Van Schaftingen, J. Asymptotics and symmetries of least energy nodal solutions of Lane-Emden problems with slow growth Communications in Contemporary Mathematics, 10/4 (2008), 609-631. DOI: 10.1142/S0219199708002910

[12] L.A Caffarelli, A. Friedman. Partial regularity of the zero-set of solutions of linear and superlinear elliptic equations, Journal of Differential Equations, 60/3 (1985), 420-433. DOI: 10.1016/00220396(85)90133-0

[13] Á. Elbert, Some recent results on the zeros of Bessel functions and orthogonal polynomials, Journal of Computational and Applied Mathematics. (2001) 133 (12), pp. 65-83. DOI: 10.1016/S03770427(00)00635-X

[14] P. Esposito, A. Pistoia, J. Wei, Concentrating solutions for the Hénon equation in $\mathbb{R}^{2}$. (2006) Journal d'Analyse Mathematique, 100, pp. 249-280. DOI: 10.1007/BF02916763

[15] B. Gidas, W.M. Ni, L. Nirenberg, Symmetry and related properties via the maximum principle. Comm. Math. Phys. 68 (1979), 209- 243.

[16] aB. GidAs AND J. Spruck , A priori bounds for positive solutions of nonlinear elliptic equations. (1981) Communications in Partial Differential Equations, 6/8, pp. 883-901. DOI 10.1080/03605308108820196

[17] F. Gladiali, A monotonicity result under symmetry and Morse index constraints in the plane, (2019) arXiv:1904.03905

[18] F. Gladiali, M. Grossi, S.L.N. Neves, Nonradial solutions for the Hénon equation in $\mathbb{R}^{N}$, Advances in Mathematics, 249 (2013), 1-36, doi:10.1016/j.aim.2013.07.022. 
[19] F. Gladiali, I. Ianni, Quasiradial nodal solutions for the Lane-Emden problem in the ball, (2017) arXiv:1709.03315

[20] M. Grossi, On the shape of solutions of an asymptotically linear problem. Ann. Sc. Norm. Sup. Pisa VIII (2009), 429-449.

[21] J. HAO, X. Chen, Y. ZhANG, Infinitely many spike solutions for the Hnon equation with critical growth, Journal of Differential Equations, 259/9 (2015), 4924-4946. DOI:10.1016/j.jde.2015.06.015.

[22] J. KüBLER, T. Weth Spectral asymptotics of radial solutions and nonradial bifurcation for the Hénon equation, (2019) arXiv:1901.00453

[23] Z. Lou, T. Weth, Z. Zhang Symmetry breaking via Morse index for equations and systems of HénonSchrodinger type, (2019) Zeitschrift fur Angewandte Mathematik und Physik, 70 (1), art. no. 35, DOI: 10.1007/s00033-019-1080-8

[24] W. M. Ni, A Nonlinear Dirichlet Problem on the Unit Ball and Its Applications. Indiana Univ. Math. J. 31 (1982), 801-807.

[25] W.M. Ni, R. D. Nussbaum, Uniqueness and nonuniqueness for positive radial solutions of $\Delta u+f(u, r)=$ 0, Comm. Pure Appl. Math. 38 (1985), 67-108.

[26] A. Pistoia, E. Serra, Multi-peak solutions for the Hénon equation with slightly subcritical growth Mathematische Zeitschrift, 256/1 (2007) , 75-97. DOI: 10.1007/s00209-006-0060-9

[27] E. Serra, Non-radial positive solutions for the Hénon equation with critical growth, Calc. Var. Partial Differ. Equ. 23, 301326 (2005)

[28] D. Smets, , J. Su, M. Willem, Non-radial ground states for the Hénon equation. Commun. Contemp. Math. 4 (2002), 467-480.

[29] J. Wei, S. YAn, Infinitely many non radial solutions for the Hénon equations with critical growth, Rev. Mat. Iberoam. 29 (2013), 997-1020.

[30] Y.-B. ZhANG, H.-T. YANG, Multi-peak nodal solutions for a two-dimensional elliptic problem with large exponent in weighted nonlinearity Acta Mathematicae Applicatae Sinica, 31/1 (2015), 261-276. DOI: $10.1007 / \mathrm{s} 10255-015-0465-5$

† Dipartimento di Scienze Applicate, Università di Napoli "Parthenope", Centro Direzionale Di NAPoli, Isola C4, 80143 NAPOLI, Italy. annalisa. Amadori@uni parthenope. it 